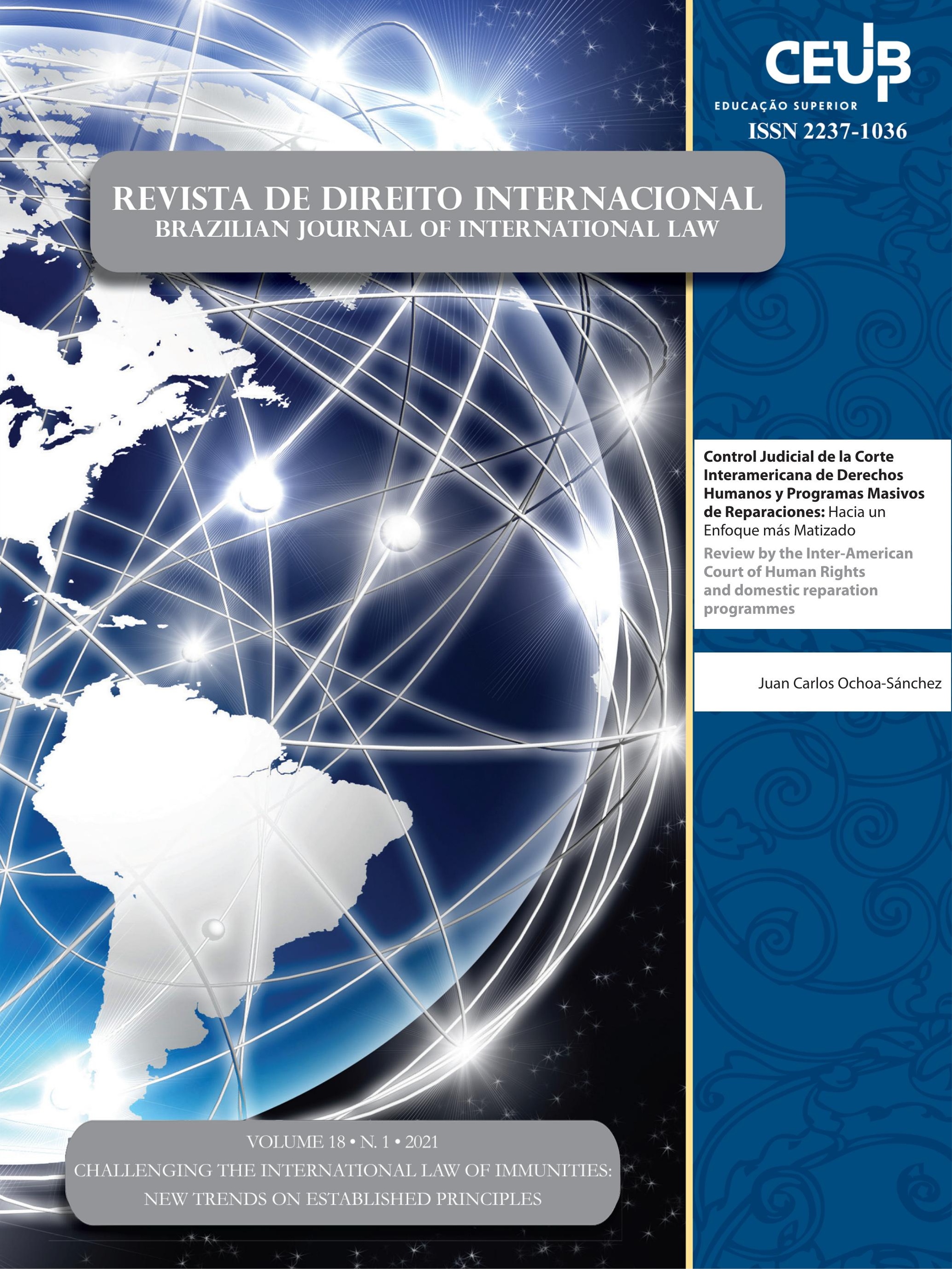


EDITORIAL

Challenging the International Law of Immunities: New Trends on Established Principles? An introduction to the special issue ............................................ 14

Lucas Carlos Lima, Loris Marotti e Paolo Palchetti

CrôniCAS........................................................................................ 17

RESPONSABILIDADE E IMUNIDADE DAS ORgANIZAÇÕES INTERNACIONAIS: PRÁTICA E DESAFIOS ...19 Vinícius Fox Drummond Cançado Trindade

The JURISDiCTIONAL IMMUNITY OF INTERNATIONAL ORgaNiZATIONS BEFORE THE BRAZILIAN SuPREMe Federal Court.

Bárbara Tuyama Sollero

The Law of State Immunity before the Brazilian Supreme Court: what is at stake WITH THE "ChANGRI-LA" CASE?

Aziz Tuffi Saliba e Lucas Carlos Lima

\section{Dossiê: Challenging the International Law of Immunities: New Trends} on Established Principles?

State Immunity and the Rights of Employees: Lights and Shadows of the Strasbourg COURT's JURISPRUdENCE .61

Pierfrancesco Rossi

A human Rights-BASEd CHALlENGE: THE KEY TO UNLOCK THE UN'S IMMUNiTY PROBLEM?...79 Héloïse Guichardaz

IMMUNITIES OF STATE OFFICIALS AND THE "FUNDAMENTALLY DIFFERENT NATURE" OF INTERNATIONAL COURTS: THE APPEALS CHAMBER DECISION IN THE JORDAN REFERRAL RE AL BASHIR ...97 Rita Guerreiro Teixeira e Hannes Verheyden 
IMUNIDADE DE JURISDIÇÃo dOS EsTADOS: O CAMINHO PARA A RELATIVIZAÇÃo

Vinícius Assis da Silveira, Luiz Felipe Costa Santana e Valesca Raizer Borges Moschen

The IMmUNity OF INTERNATIONAL ORganizations IN LABOUR DispUTES. DeVElopments BEFORE INTERNATIONAL TRIBUNALS, NATIONAL COURTS AND THE COLOMBIAN JURISDICTION ..... 137 Walter Arévalo-Ramirez e Ricardo Abello-Galvis

Imunidade de Jurisdição dos Estados E Poder Executivo brasileiro: os Pareceres dos CONSULTORES JURÍDICOS DO ITAMARATY

George Rodrigo Bandeira Galindo

Artigos SOBRe outros temas

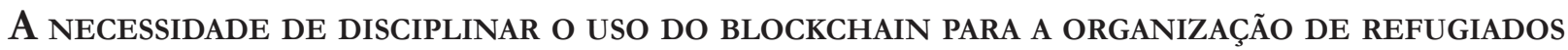
PELO DIREITO INTERNACIONAL. 195

Agatha Gonçalves Santana, Carla Noura Teixeira e Otavio Noura Teixeira

O TRABALHO INFANTIL DE MIGRANTES E REFUgIADOS VENEZUELANOS NO BRASIL

André Viana Custódio e ohana Cabral

THE NEW BRAZILIAN ANTI-TRAFFICKING LAW: CHALLENGES AND OPPORTUNITIES TO COVER THE NORMATIVE LACK . 243 Waldimeiry Correa da Silva

¿SON PARTE DEL BLOQUE DE CONSTITUCIONALIDAD LOS TRATADOS INTERNACIONALES DE DERECHOS HuMANOS DE LA OEA EN CHILE?: AVANCES EN BASE A LA DOCTRINA, NORMATIVA Y JURISPRUDÊNCIA. 270

Juan Pablo Díaz Fuenzalida

O Tratado de Saint-Germain-En-Laye e os Efeitos do instituto da “opÇão” Sobre a CONDIÇÃO JURÍDICA DOS DESCENDENTES DE CIDADÃOS AUSTRÍACOS TRENTINO-TIROLÊSES ......290 Arno Dal Ri Jr. e Andrey José Taffner Fraga

Control Judicial de la Corte Interamericana de Derechos Humanos y Programas Masivos de Reparaciones: Hacia un Enfoque más Matizado .309 Juan Carlos Ochoa-Sánchez 
JuSTICIABILIDADE DIRETA DOS DIREITOS ECONÔMICOS, SOCIAIS, CULTURAIS E AMBIENTAIS NA

Corte Interamericana DE Direitos Humanos..........................................................334

Augusto Antônio Fontanive Leal e Guilherme Massaú

How the indigenous case of Xukuru before the Inter-American Court of Human RiGHTS CAN INSPIRE DECOLONIAL COMPARATIVE STUDIES ON PROPERTY RIGHTS..........................353

Flavianne Fernanda Bitencourt Nóbrega e Camilla Montanha 


\title{
Control Judicial de la Corte Interamericana de Derechos Humanos y Programas Masivos de Reparaciones: Hacia un Enfoque más Matizado*
}

\author{
Review by the Inter-American Court of \\ Human Rights and domestic reparation \\ programmes
}

Juan Carlos Ochoa-Sánchez ${ }^{* *}$

\section{Resumen}

Este artículo aporta nuevas perspectivas a la pregunta: ¿qué tipo de control judicial debe ejercer la Corte Interamericana de Derechos Humanos (CtIDH) en casos en contra de un Estado en el cual se ha implementado un programa masivo de reparaciones (PMR)? Particularmente, este contribuye a responder la pregunta: ¿qué tipo de ordenes de reparaciones debe adoptar esta Corte en dichos casos? Con el fin de alcanzar ese objetivo general, este artículo tiene dos objetivos específicos. Primero, este trabajo analiza críticamente el punto de vista según el cual cuando la CtIDH ordena reparaciones en este tipo de casos debe necesariamente centrar su decisión sólo en las violaciones de los derechos humanos encontradas en el caso individual que se le presenta. Segundo, este trabajo propone un enfoque en el que la CtIDH examina las características de los PMR aplicables a los casos individuales presentados ante este Tribunal. Particularmente, la CtIDH debe evaluar si un determinado PMR cumple con las obligaciones del Estado en virtud de la Convención Americana de Derechos Humanos. Este trabajo utiliza un método cualitativo y cubre la perspectiva analítica, la de derecho internacional, la de derecho constitucional comparado y la normativa. Este artículo demuestra que el argumento de que la $\mathrm{CtIDH}$ debe limitarse a un enfoque de caso individual en el tipo de casos analizados es injustificado desde una perspectiva jurídica, analítica y normativa. La práctica internacional en materia de derechos humanos, la propia jurisprudencia de la CtIDH y la práctica constitucional comparada, especialmente respecto a formas generales de control judicial por parte de tribunales de derecho público, proveen los principales fundamentos jurídicos de la tesis expuesta y del enfoque propuesto en este artículo.

Palabras claves: Reparaciones. Programas nacionales de reparación. Justicia transicional. Formas generales de control judicial. Sistema Interamericano de Derechos Humanos.

** Juan Carlos Ochoa-Sánchez holds a Ph.D. in International Relations, Specialization in International Law, from the University of Geneva, the Graduate Institute of International and Development Studies in Geneva, Switzerland. He holds an LL.M. from Leiden University in the Netherlands. He is an Associate Professor at Universidad Católica de Colombia, Bogotá. He has worked at the University of Oslo (Norway), the International Labour Organization, and the United Nations.

E-mail: juancarlos.ochoasanchez@graduateinstitute.ch 


\section{Abstract}

This article brings new perspectives to the question of which type of review the Inter-American Court of Human Rights (IACtHR) should adopt for remedies in cases involving a State where a Domestic Reparation Programme (DRP) has been implemented. Particularly, it contributes to answering the question: ¿which type of remedies should adopt this Court in these cases? To meet this general objective, this paper has two specific objectives. First, it critically examines the view that posits that when the IACtHR orders remedies in the examined cases it should focus its decision on only the discrete human rights violations found in an individual case before it. Second, this article proposes an approach where the IACtHR reviews the characteristics of the DRPs applicable to individual cases brought before it. Notably, this Court should evaluate whether a given DRP meets State obligations under the American Convention on Human Rights (ACHR). This paper uses a qualitative method and covers the international law, analytical, comparative constitutional law, and normative perspective. This article demonstrates that the argument that the IACtHR should limit itself to an individual case approach to remedies in the examined cases is unwarranted from a legal, analytical, and normative perspective. International human rights practice, the jurisprudence of the Inter-American Court of Human Rights and comparative constitutional practice, especially on general forms of judicial review by public law courts, provide the main legal bases for the thesis put forward and the approach proposed in this article.

Keywords: Reparations. Domestic reparation programmes. Remedies. Transitional justice. General forms of judicial review. Inter-American Human Rights System

\section{Introducción}

Los países de América Latina y otras regiones del mundo han adoptado cada vez más programas masivos de reparaciones (PMR), como parte de sus esfuerzos para hacer frente a violaciones de los derechos humanos perpetradas en gran escala durante un conflicto armado o situación de represión. Como lo menciona Fabian Salvioli, el Relator Especial de la Organización de Naciones Unidas (ONU) sobre la promoción de la verdad, la justicia, la reparación, y las garantías de no reparación, las principales características de dichos programas son las siguientes:

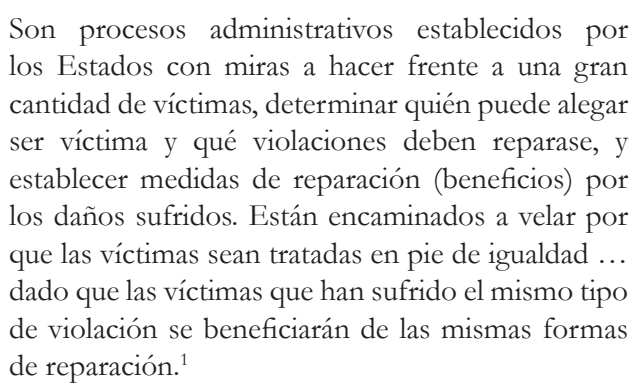

Adicionalmente, los PMR proporcionan reparaciones mediante un proceso sumario y sin la elevada carga y el estándar de prueba que se requieren en los procesos judiciales. $^{2}$

Además de las ventajas logísticas que los PMR tienen en comparación con reparaciones ordenadas por cortes nacionales, como se puede ver estos programas tienen un alcance general en dos importantes sentidos: En primer lugar, estos programas cubren a todas las víctimas que cumplen los criterios establecidos en dichos programas. En segundo lugar, estos programas usualmente establecen que deben aplicarse los mismos estándares de reparación a todas las víctimas que se encuentren en la misma situación en el país en cuestión y que estén cubiertas por el PMR específico. ${ }^{3}$ Dado el alcance general de los PMR, las características que usualmente tienen las sociedades en transición y la importancia de

1 Fabian Salvioli, Informe del Relator Especial sobre la promoción de la verdad, la justicia, la reparación, y las garantías de no reparación, 11 de julio de 2019, documento. de la ONU A/HRC/42/45, párrafo 31 (en adelante 'Salvioli, doc. de la ONU A/HRC/42/45').

${ }_{2}$ Ver por ejemplo SANDOVAL, Clara. 'Two Steps Forward, One Step Back: Reflections on the Jurisprudential Turn of the InterAmerican Court of Human Righs on Domestic Reparation Programmes'. The International Journal of Human Rights 22, n. 9, p. 1193, 2018. Ver también CORREA, Cristian. 'Reparations for Victims of Massive Crimes. Making Concrete a Message of Inclusion'. En: Victimological Approaches to International Crimes: Africa. LETSCHERT, Rianne et al. (ed.). Cambridge: Antwerp and Portland: Intersentia, 2011. p. 203; DE GREIFF, Pablo. Informe del Relator Especial sobre la promoción de la verdad, la justica, la reparación y las garantías de no repetición, 14 de octubre de 2014, doc. de la ONU A/69/518, 3 (en adelante "informe de Pablo de Greiff de 2014"); GARCÍA-GODOS, Jemima. 'Victim Reparations in Transitional Justice - What is at Stake and Why'. Nordic Journal of Human Rights, v. 26, n. 2, 2008.

3 Ver por ejemplo, SALVIOLI, Fabian. Informe del Relator Especial sobre la promoción de la verdad, la justicia, la reparación, y las garantías de no reparación, 11 de julio de 2019, documento. de la ONU A/HRC/42/45, párrafo 31. 
aplicar estándares de reparación iguales a todas las víctimas en la misma situación en el país en cuestión, estos programas son medidas que potencialmente podrían ser muy importantes para proporcionar reparaciones en estas sociedades. ${ }^{4}$ Los contextos de transición usualmente involucran un gran número de violaciones de los derechos humanos y de víctimas, y los gobiernos de estas sociedades se enfrentan a importantes restricciones financieras para satisfacer las numerosas necesidades que dichas sociedades tienen.

Desde junio de 2013, la Corte Interamericana de Derechos Humanos (CtIDH) ha decidido casos relativos a los PMR establecidos en cuatro países de América Latina: Chile, Colombia, Guatemala y Perú. ${ }^{5}$ Ha surgido entonces la pregunta fundamental: ¿qué tipo de control judicial debe ejercer la CtIDH en casos en contra de un Estado en el cual se ha implementado un PMR? Específicamente, ¿qué tipo de ordenes de reparaciones debe adoptar la CtIDH en dichos casos? La mejor manera de ilustrar estas preguntas es con un ejemplo de la vida real. Durante el conflicto armado interno que hubo en Guatemala entre 1980 y 1996, violaciones masivas a los derechos humanos fueron perpetradas. ${ }^{6}$ Según la Comisión de la Verdad de Guatemala, miembros de las Fuerzas Armadas perpetraron 626 masacres durante ese conflicto armado. ${ }^{7}$ En 2003, se estableció en Guatemala un PMR para reparar a las víctimas de violaciones de los derechos humanos durante ese conflicto. ${ }^{8}$ En 2004, la CtIDH decidió el caso de la masacre de Plan de Sánchez c. Guatemala. Se trata de la masacre de aproximadamente 268 personas, la mayoría de ellas miembros del pueblo indígena Maya Achí; y el saqueo y destrucción de las viviendas de los habitantes del pueblo de Plan de Sánchez

\footnotetext{
${ }^{4}$ Ver por ejemplo SALVIOLI, Fabian. Informe del Relator Especial sobre la promoción de la verdad, la justicia, la reparación, y las garantías de no reparación, 11 de julio de 2019, documento. de la ONU A/HRC/42/45, párrafos 31, 32, 34.

5 Este artículo analiza abajo las decisiones relevantes de la CtIDH. Ver la sección denominada "Principales enfoques adoptados por la Corte Interamericana."

${ }^{6}$ Para un análisis, ver por ejemplo, GARCÍA-GODOS, Jemima; SALVADÓ, Luis Raúl. Guatemala: Truth and memory on trial. En: SKAAR, E.; GARCIA-GODOS, J.; COLLINS, C. (ed.) Transitional Justice in Latin America. Londres: Routledge, 2016.

GOBIERNO DE GUATEMALA. Comisión para el Esclarecimiento Histórico (1999). Guatemala: Memoria del Silencio, Conclusiones y Recomendaciones (Ciudad de Guatemala: Comisión para el Esclarecimiento Histórico), párrafo 86.

8 Este PMR fue creado por un acto jurídico del Ejecutivo - i.e. Acuerdo Gubernativo 258-2003, y ha sido reformado por otros Acuerdos Gubernativos como el 539-2013.
}

y pueblos vecinos, entre otras violaciones a los derechos humanos. ${ }^{9}$ Guatemala solicitó a la CtIDH que esta última se remitiera al PMR de Guatemala en su decisión sobre reparaciones..$^{10}$ Este programa establecía reparaciones menos integrales y costosas que las ordenadas usualmente por la CtIDH. La CtIDH determinó que se violaron los derechos a la vida, al trato humano y a la propiedad, entre otros derechos. ${ }^{11}$

En este tipo de contexto, la CtIDH debe responder preguntas tales como: ¿tiene esta Corte alguna flexibilidad al diseñar y dar órdenes de reparación en este tipo de casos? Concretamente, ¿podría la CtIDH concentrarse primero en evaluar si el PMR, que se aplica a un caso ante esta Corte, cumple con las obligaciones del Estado bajo la Convención Americana sobre Derechos Humanos? Si se cumplen estas obligaciones, ¿podría la CtIDH remitirse a lo que establece un determinado PMR en su decisión sobre reparaciones? Dado que los PMR proporcionan medidas generales de reparación, este tipo de control por parte de la CtIDH tendría un alcance general. Por lo tanto, la CtIDH ejercería lo que en la práctica constitucional comparada se conoce como "una forma general de control judicial." En formas generales de control judicial, una Corte usa procedimientos que buscan la adopción de medidas generales para resolver problemas que causan un patrón de violaciones a los derechos humanos o abusos repetitivos. ${ }^{12}$

La CtIDH debe abordar una pregunta adicional: cuando este Tribunal ordena reparaciones en este tipo de casos, ¿debe necesariamente centrar su decisión sólo en las violaciones de los derechos humanos encontra-

\footnotetext{
9 CTIDH. caso de la masacre de Plan de Sánchez contra Guatemala, sentencia de fondo, 29 de abril de 2004, párrafo 42.21; CTIDH. caso de la masacre de Plan de Sánchez contra Guatemala, sentencia de reparaciones, 19 de noviembre de 2004, párrafo 49.4.

10 PRESIDENCIA DE LA REPUBLICA DE GUATEMALA. COPREDEH, 21 de mayo de 2004, RED-A-28-2004, punto 7, 15.

${ }^{11}$ CTIDH. caso de la masacre de Plan de Sánchez contra Guatemala, sentencia de fondo, 29 de abril de 2004, párrafos 47, 52.

12 Malcolm Langford describe los principales elementos de formas generales de control judicial. Según este autor, en estas formas de control, un tribunal examina un patrón de violaciones de los derechos humanos o abusos repetitivos causados por problemáticas comunes, y utiliza procedimientos que tienen por objeto adoptar medidas generales para abordar esas problemáticas. Ver LANGFORD, Malcolm. 'Social Rights Adjudication. An Interdisciplinary Perspective'. Tesis (Doctorado) Facultad de Derecho, Universidad de Oslo, 2014. p. 184, 192-304. Ver también RODRÍGUEZ-GARAVITO, César; RODRÍGUEZ-FRANCO, Diana. Juicio a La Exclusión: El Impacto de los Tribunales sobre los Derechos Sociales en el Sur Global. Buenos Aires: Siglo 21 Editora Iberoamericana, 2015.
} 
das en el caso individual que se le presenta? Este enfoque puede denominarse "una forma de control judicial limitada a casos individuales" o "un enfoque de caso individual". El alcance de esta forma de control judicial es muy diferente al alcance de una forma general de control judicial. Igualmente, como se analizará más adelante, estas dos formas de control judicial llevarían a resultados muy diferentes en términos de derecho internacional y normativos.

En la mayoría de sus decisiones dictadas entre junio de 2013 y noviembre de 2018, la CtIDH ha adoptado un enfoque de caso individual respecto a sus órdenes de reparaciones en este tipo de casos. De igual manera, los trabajos académicos existentes sobre este tema han defendido implícitamente un enfoque de caso individual respecto a las órdenes de reparación que debe dictar la CtIDH en este tipo de casos. ${ }^{13}$ Como se examinará más adelante, el principio de la reparación integral ha sido considerado por la CtIDH y académicos como un fundamento clave para un enfoque de caso individual en el tipo de casos estudiados. Sin embargo, estos trabajos académicos y la CtIDH no han analizado las serias dificultades jurídicas y normativas que plantea un enfoque de caso individual en los casos estudiados. ${ }^{14}$ Por ejemplo, un número significativo de expertos internacionales en derechos humanos ha sostenido que el principio de la reparación integral se elaboró partiendo del supuesto de que lo que se pretende es la resolución de casos relativamente aislados, y no de violaciones de los derechos humanos cometidas en gran escala como las que se abordan en contextos de transición. ${ }^{15}$

\footnotetext{
${ }^{13}$ Ver por ejemplo, SANDOVAL, Clara. 'Two Steps Forward, One Step Back: Reflections on the Jurisprudential Turn of the InterAmerican Court of Human Righs on Domestic Reparation Programmes'. The International Journal of Human Rights, 22, n. 9, p. 11931204, 2018. Si bien Geneviève Lessard parece hacer diferenciaciones según la forma de reparación de que se trate, ella defiende implícitamente un enfoque de caso individual de la CtIDH en el tipo de casos examinados, al menos en lo que respecta a indemnización. Ver GENEVIÈVE, Lessard. 'Preventive Reparations at a Crossroads: The Inter-American Court of Human Rights and Colombia's Search for Peace'. The International Journal of Human Rights, v. 22, n. 9, p. 1221, 2018. Para un análisis completo de este aspecto, ver abajo la sección "Posiciones adoptadas en estudios existentes."

14 Ver SANDOVAL, Clara. 'Two Steps Forward, One Step Back: Reflections on the Jurisprudential Turn of the Inter-American Court of Human Righs on Domestic Reparation Programmes'. The International Journal of Human Rights, 22, n. 9, 2018; Lessard, 'Preventive Reparations at a Crossroads'.

15 Ver por ejemplo, DE GREIFF, Pablo. 'Justice and Reparations', en The Handbook of Reparations. Oxford: Oxford University Press, 2006. p. 454. Para un análisis completo de esta crítica, ver abajo la
}

Además, las investigaciones existentes sobre las reparaciones ordenadas por la CtIDH en el tipo de casos examinados no han analizado rigurosamente la posibilidad de que esta Corte evalúe si un PMR, aplicable a un caso que se le presenta, cumple con las obligaciones del Estado en virtud de la Convención Americana sobre Derechos Humanos. ${ }^{16}$ Esta omisión es preocupante. En primer lugar, la práctica internacional en materia de derechos humanos, la propia jurisprudencia de la CtIDH y la práctica constitucional comparada demuestran que la CtIDH puede ejercer por lo menos algunas formas generales de control judicial, en particular respecto a ordenes de reparaciones. ${ }^{17}$ En segundo lugar, como se analizará más adelante en este artículo, la CtIDH ejercería en el tipo de casos analizados una forma general de control judicial relativamente limitada.

Este artículo busca aportar nuevas perspectivas a la pregunta: ¿qué tipo de control judicial debe ejercer la CtIDH en casos en contra de un Estado en el cual se ha implementado un PMR? Particularmente, este busca contribuir a responder a la pregunta: ¿qué tipo de ordenes de reparaciones debe adoptar la CtIDH en estos casos? Con el fin de alcanzar ese objetivo general, este trabajo tiene dos objetivos específicos. El primero es analizar críticamente el punto de vista según el cual cuando la CtIDH ordena reparaciones en este tipo de casos debe necesariamente centrar su decisión sólo en las violaciones de los derechos humanos encontradas en el caso individual que se le presenta. Este artículo demostrará que el argumento de que la CtIDH debe limitarse a un enfoque de caso individual en el tipo de casos examinados es injustificado desde una perspectiva jurídica, analítica y normativa.

Segundo, este trabajo busca proponer un enfoque más matizado respecto a las órdenes de reparaciones que la CtIDH debe adoptar en el tipo de casos estudiados que el de caso individual y otros enfoques que han

sección titulada "Un enfoque de caso individual: Un análisis crítico", subsección "¿Debe aplicarse el principio de la reparación integral en contextos de transición?"

${ }^{16}$ Ver SANDOVAL, Clara. 'Two Steps Forward, One Step Back: Reflections on the Jurisprudential Turn of the Inter-American Court of Human Righs on Domestic Reparation Programmes'. The International Journal of Human Rights, 22, n. 9, 2018; Lessard, 'Preventive Reparations at a Crossroads'.

17 Para un análisis completo de este punto, ver abajo la sección titulada "Un enfoque de caso individual: Un análisis crítico", subsección "Limitarse a un enfoque de caso individual en los casos estudiados es injustificado teniendo en cuenta los poderes de la Corte Interamericana.' 
sido propuestos. Este propone un enfoque en el que la CtIDH examina las características de los PMR aplicables a los casos individuales presentados ante este Tribunal. En especial, la CtIDH debe evaluar si un determinado PMR cumple con las obligaciones del Estado en virtud de la Convención Americana de Derechos Humanos. Este artículo demostrará que la práctica internacional de los derechos humanos, la propia jurisprudencia de la CtIDH y la práctica constitucional comparada proveen fundamento para el enfoque que se propone en este trabajo. También demostrará que el enfoque propuesto responde bien a principios normativos relevantes.

Este trabajo utiliza un método cualitativo y cubre la perspectiva analítica, la de derecho internacional, la de derecho constitucional comparado y la normativa. En particular, las principales tesis de este artículo se fundamentarán en aportes analíticos y desarrollos recientes en la práctica internacional en derechos humanos y en la adjudicación comparada en materia de derechos fundamentales, especialmente respecto a formas generales de control judicial por parte de tribunales de derecho público. ${ }^{18}$ Estas tesis se basan también en aportes analíticos claves de la literatura sobre reparaciones en contextos de transición, incluyendo en países en donde se han implementado un PMR y la CtIDH ha conocido uno o más casos en contra de uno de dichos países. ${ }^{19}$ Debe clarificarse que la perspectiva normativa se refiere a cuestiones más amplias que aquellas recogidas en lex lata y que son importantes a la luz de principios democráticos bien establecidos en sistemas democráticos.

En lo que sigue de este artículo, primero se examinaran las principales características de los PMR. Segundo, se analizarán las posiciones sobre la cuestión analizada adoptadas en las investigaciones existentes. Tercero, se estudiarán las principales posiciones adoptadas por la CtIDH sobre la cuestión examinada en su jurispruden-

18 Ver por ejemplo, Langford, 'Social Rights Adjudication', 184, 192-304; Rodríguez-Garavito y Rodríguez-Franco, Juicio a La Exclusión; Nino Tsereteli, 'The Role of the European Court of Human Rights in Facilitating Legislative Change in Cases of Long-Term Delays in Implementation', en The International Human Rights Judiciary and National Parliaments: Europe and Beyond, Studies on Human Rights Conventions, eds. Andreas Føllesdal, Geir Ulfstein, y Matthew Saul (Cambridge: Cambridge University Press, 2017).

19 Ver por ejemplo, MERSKY, Marcie; ROHT-ARRIAZA, Naomi. 'Guatemala'. En: SALAZAR, Katya; ANTKOWIAK,Thomas. (ed.). Victims Unsilenced: The Inter-American Human Rights System and Transitional Justice in Latin America. Washington, DC: Due Process of Law Foundation, 2007. p. 19-20, 29-30; Pablo de Greiff, 'Justice and Reparations'. cia. Cuarto, se examinará críticamente el punto de vista según el cual cuando la CtIDH ordena reparaciones en los casos examinados, debe necesariamente adoptar un enfoque de caso individual. Quinto, se propondrá un enfoque alternativo para responder de manera satisfactoria a las dificultades jurídicas y normativas que plantea un enfoque de caso individual. Por último, se presentarán las principales conclusiones y argumentos de este trabajo.

\section{Principales características de los PMR}

Primero, debe mencionarse que varias de las características generales de los PMR fueron mencionadas previamente en la introducción, tales como su alcance general y que estos proveen medidas de reparación mediante un proceso sumario y sin la elevada carga y el estándar de prueba que se requieren en un entorno judicial. Segundo, debe agregarse que según los Principios y directrices básicos sobre el derecho de las víctimas de violaciones manifiestas de las normas internacionales de derechos humanos y de violaciones graves del derecho internacional humanitario a interponer recursos y obtener reparaciones existen cinco formas de reparación: 1) restitución, 2) indemnización, 3) rehabilitación, 4) satisfacción y 5) garantías de no repetición. ${ }^{20}$ Tradicionalmente, los PMR se han centrado en indemnización, rehabilitación y restitución. ${ }^{21}$ Sin embargo, algunos PMR adoptados en América Latina en la última década han sido más completos. Además de indemnización, rehabilitación y restitución, abarcan ciertas formas de satisfacción. Específicamente, estos abarcan ciertas medidas de reparación simbólica que están directamente relacionadas con la búsqueda y el reconocimiento de la verdad. Ejemplos de estas medidas son las disculpas oficiales, la creación de museos y parques dedicados a

\footnotetext{
20 Principios y directrices básicos sobre el derecho de las víctimas de violaciones manifiestas de las normas internacionales de derechos humanos y de violaciones graves del derecho internacional humanitario a interponer recursos y obtener reparaciones, Asamblea General de la ONU, Res. 60/147, 16 de diciembre 2005, doc. de la ONU A/ RES/60/147 (en adelante "Los Principios y directrices básicos de Naciones Unidas sobre el derecho a interponer recursos y obtener reparaciones").

${ }_{21}$ Ver informe de DE GREIFF, Pablo. Informe del Relator Especial sobre la promoción de la verdad, la justica, la reparación y las garantías de no repetición, 14 de octubre de 2014, doc. de la ONU A/69/518, párrafos 19, 21.
} 
la memoria de las víctimas, el cambio de nombre de los espacios públicos y la restauración del buen nombre de las víctimas. ${ }^{22}$ Algunos de estos PMR incluyen también garantías de no repetición. Este es el caso del PMR de Colombia. ${ }^{23}$

Tercero, actualmente órganos y expertos internacionales subrayan que las indemnizaciones individuales concedidas como parte de estos programas deben ir acompañadas del reconocimiento de responsabilidad y otras medidas para buscar verdad, justicia y garantías de no repetición. ${ }^{24} \mathrm{La} \mathrm{CtIDH}$ ha afirmado este requisito en su jurisprudencia. ${ }^{25}$ El Relator Especial de las Naciones Unidas, el profesor Salvioli, también ha incluido esto entre los requisitos mínimos que los PMR deben cumplir. ${ }^{26} \mathrm{El}$ fundamento de este requisito es evitar que las indemnizaciones se consideren como un pago a cambio del silencio o la aquiescencia de las víctimas y sus familias. ${ }^{27}$

Otra característica de estos programas es que las reparaciones individuales que conceden no se basan en el principio de la reparación integral. Pablo de Greiff

22 Para un estudio de la práctica de los Estados respecto a PMR, ver Salvioli, doc. de la ONU A/HRC/42/45. Para análisis por parte de académicos, ver Sandoval, 'Two Steps Forward, One Step Back'; informe de Pablo de DE GREIFF, Pablo. Informe del Relator Especial sobre la promoción de la verdad, la justica, la reparación y las garantías de no repetición, 14 de octubre de 2014, doc. de la ONU A/69/518. p. 9. Para una enumeración ilustrativa de medidas de satisfacción, ver los Principios y directrices básicos de Naciones Unidas sobre el derecho a interponer recursos y obtener reparaciones, Principio 22.

23 CONGRESO DE COLOMBIA. Ley de Victimas y Restitución de Tierras, Ley 1448/2011. https://www.unidadvictimas.gov.co/sites/ default/files/documentosbiblioteca/ley-1448-de-2011.pdf

${ }^{24}$ Informe de DE GREIFF, Pablo. Informe del Relator Especial sobre la promoción de la verdad, la justica, la reparación y las garantías de no repetición, 14 de octubre de 2014, doc. de la ONU A/69/518, párrafos 11, 83. Para puntos de vistas por parte de académicos, ver MOFFETT, L. Transitional Justice and Reparations: Remedying the Past? In: LAWTHER, C.; MOFFETT, L.; JACOBS, D. (ed.). Research Handbook on Transitional Justice. Edward Elgar, 2017; BORNEMAN, John. Political Crime and the Memory of Loss. Indiana University Press, 2011. p. 11.

25 Ver por ejemplo, CTIDH. Comunidades afrodescendientes desplazadas de la cuenca del río Cacarica (Operación Génesis) vs. Colombia, sentencia de 22 de noviembre de 2016, párrafo 326 (en adelante 'Operación Génesis contra Colombia).

26 Salvioli, doc. de la ONU A/HRC/42/45, párrafo 44(d).

${ }^{27}$ MOON, Claire. 'Who'll Pay Reparations on My Soul?'1 Compensation, Social Control and Social Suffering'. Social \& Legal Studies 21, n. 2, 2012. p. 4-5, 10; Informe de Pablo de Greiff de 2014, párrafo 11; MOFFETT, L. Transitional Justice and Reparations: Remedying the Past? In: LAWTHER, C.; MOFFET'T, L.; JACOBS, D. (ed.). Research Handbook on Transitional Justice. Edward Elgar, 2017. p. 23. informa: "La experiencia con los programas masivos de reparación sugiere... que rara vez se intenta satisfacer este criterio." ${ }^{28} \mathrm{El}$ contenido de la obligación de reparación según el principio de la reparación integral puede definirse de la siguiente manera: La reparación debe, en la medida de lo posible, eliminar todas las consecuencias del acto ilícito y restablecer la situación que, con toda probabilidad, habría existido si ese acto no se hubiera cometido. ${ }^{29}$

Esta definición fue adoptada por la Corte Permanente de Justicia Internacional en su decisión en Factory at Chorzón, Alemania contra Polonia de 1928. Este dicta ha sido reiterado en muchas decisiones de cortes internacionales. Entonces, esta es una diferencia importante entre las reparaciones individuales concedidas por PMR y las ordenadas por tribunales tradicionalmente. Estas últimas se basan en el principio de la reparación integral. Mas adelante se analizará esta diferencia y sus efectos.

Por último, respecto al uso de los PMR, el Relator Especial de las Naciones Unidas, el profesor Salvioli, informa lo siguiente:

Estos programas se han utilizado en diversas partes del mundo. Han abordado las atrocidades cometidas durante represión, como en la Argentina, Chile, Iraq o Marruecos, o durante conflictos, como en Bosnia y Herzegovina, Colombia, Guatemala, Iraq y Sierra Leona. ${ }^{30}$

\section{Posiciones adoptadas en estudios existentes}

Estudios existentes sobre la cuestión analizada han contrastado el enfoque de la CtIDH en sus decisiones sobre PMR dictadas entre noviembre de 2013 y octubre de 2016 con el enfoque respecto a reparaciones adoptado por esta Corte en la gran mayoría de casos con-

\footnotetext{
${ }^{28}$ Ver informe de DE GREIFF, Pablo. Informe del Relator Especial sobre la promoción de la verdad, la justica, la reparación y las garantías de no repetición, 14 de octubre de 2014, doc. de la ONU A/69/518, párrafo 45. Ver también MALAMUD-GOTI, Jaime E.; GROSMAN, Lucas Sebastián; DE GREIFF, Pablo. 'Reparations and Civil Litigation: Compensation for Human Rights Violations inTransitional Democracies'. En: DE GREIFF, Pablo. (ed.). The Handbook of Reparations. Oxford: Oxford University Press, 2006.

29 TRIBUNAL PERMANENTE DE JUSTICIA INTERNACIONAL. Factory at Chorzón, Alemania c. Polonia, reclamación por indemnización, sentencia No 13, (1928) PCIJ Series A No 17, ICGJ 255 (PCIJ 1928), 13 septiembre 1928, párrafo 47.

30 Salvioli, doc. de la ONU A/HRC/42/45, párrafo 33.
} 
tenciosos. Cabe señalar que el enfoque adoptado por la CtIDH respecto a reparaciones en la mayoría de los casos contenciosos se ha basado en el principio de la reparación integral. ${ }^{31}$ Sin embargo, como se analizará, durante el período comprendido entre noviembre de 2013 y octubre de 2016 la CtIDH se remitió a lo que establecen los PMR pertinentes sin exigir el cumplimiento de requisitos significativos. ${ }^{32}$ En este respecto, Clara Sandoval argumenta que:

\section{El tratamiento de la subsidiariedad [de la CtIDH] y su cambiante jurisprudencia sobre los PMR constituye un paso atrás, ya que están transformando los estándares interamericanos, tanto sustantivos como procesales, en materia de reparación sin argumentos jurídicos suficientes. ${ }^{33}$}

Clara Sandoval también afirma: "El cambio jurisprudencial [de la $\mathrm{CtIDH}$ ] impide que las víctimas puedan obtener una reparación adecuada, rápida y efectiva del daño sufrido". ${ }^{34}$ De los argumentos dados por Sandoval para apoyar esta y otras afirmaciones en el citado artículo, parece desprenderse que ella asume que, de acuerdo con el derecho internacional de los derechos humanos, el principio de la reparación integral debe necesariamente ser aplicado por la CtIDH en un caso cubierto por un PMR. Esto a pesar de las características antes mencionadas de los escenarios de transición. Aunque ella no dice esto explícitamente, este supuesto parece estar implícito en varios de sus argumentos. Por ejemplo, en la sección de conclusiones, ella dice que una cuestión fundamental sobre la cual la CtIDH "todavía tiene que articular argumentos jurídicos adecuados y abordar... es si los estándares jurídicos aplicados por los PMR son compatibles o no con el derecho internacional de los

\footnotetext{
31 Ver por ejemplo CTIDH. Velasquez Rodriguez vs. Honduras, sentencia de reparaciones, 21 de julio de 1989, párrafo 26; CTIDH. Juan Humberto Sánchez vs. Honduras, sentencia de 7 de junio de 2003, párrafo 149. Para análisis por parte de académicos, ver por ejemplo, Sandoval, 'Two Steps Forward, One Step Back'; Thomas M. Antkowiak, 'Remedial Approaches to Human Rights Violations: The Inter-American Court of Human Rights and Beyond', Columbia Journal of Transnational Law, 46, no. 2 (2008).

32 Ver por ejemplo, CTIDH. Operación Génesis vs. Colombia, sentencia de 20 noviembre de 2013; CTIDH. caso de Yarce y otros vs. Colombia, sentencia de objeciones preliminares, fondo, reparaciones y costos, 22 de noviembre de 2016, párrafo 326.

33 SANDOVAL, Clara. 'Two Steps Forward, One Step Back: Reflections on the Jurisprudential Turn of the Inter-American Court of Human Righs on Domestic Reparation Programmes'. The International Journal of Human Rights, 22, n. 9, p. 1204, 2018.

34 SANDOVAL, Clara. 'Two Steps Forward, One Step Back: Reflections on the Jurisprudential Turn of the Inter-American Court of Human Righs on Domestic Reparation Programmes'. The International Journal of Human Rights, 22, n. 9, p. 1203, 2018.
}

derechos humanos ..." ${ }^{35}$ Otros autores que han escrito sobre el tema analizado adoptan la misma premisa. ${ }^{36}$

Como se señaló anteriormente, las reparaciones individuales que conceden los PMR no se basan en el principio de la reparación integral. Entonces, una implicación lógica de la premisa que estos autores parecen adoptar es que la CtIDH debe necesariamente rechazar toda solicitud de remisión a lo que establece un determinado PMR en su decisión sobre reparaciones. En consecuencia, en los casos examinados la CtIDH siempre tendría que centrar su decisión sobre reparaciones únicamente en las violaciones de los derechos humanos encontradas en el caso individual que se le presente. En conclusión, puede decirse que, al adoptar la premisa mencionada, estos autores han adoptado implícitamente un enfoque de caso individual en relación con las ordenes de reparaciones que la CtIDH debe adoptar en los casos analizados. Otros elementos de la obra de estos autores apuntan a la misma conclusión. ${ }^{37}$

Además, estudios existentes sobre la temática analizada no han examinado de manera exhaustiva la posibilidad de que la CtIDH evalúe si un PMR, aplicable a un caso ante la Corte, cumple con las obligaciones del Estado en virtud de la Convención Americana sobre Derechos Humanos. Como se explicará más adelante, esta omisión es preocupante por varias razones.

El presente artículo hace aportes significativos a los trabajos académicos existentes sobre este y otros temas más amplios. Primero, la presente investigación demos-

35 SANDOVAL, Clara. 'Two Steps Forward, One Step Back: Reflections on the Jurisprudential Turn of the Inter-American Court of Human Righs on Domestic Reparation Programmes'. The International Journal of Human Rights, 22, n. 9, p. 1204, 2018. Ver también ibid., 1200-1201, y 1203-1204. Digo "parece" porque la posición de Sandoval sobre este punto no es suficientemente clara en su artículo. ${ }^{36}$ Lessard adopta la misma premisa por lo menos respecto a indemnización. Ver GENEVIÈVE, Lessard. 'Preventive Reparations at a Crossroads: The Inter-American Court of Human Rights and Colombia's Search for Peace'. The International Journal of Human Rights, v. 22 , n. 9, p. 1221, 2018.

37 Ver por ejemplo, SANDOVAL, Clara. 'Two Steps Forward, One Step Back: Reflections on the Jurisprudential Turn of the Inter-American Court of Human Righs on Domestic Reparation Programmes'. The International Journal of Human Rights, 22, n. 9, p. 1200-1201, 2018 y 1203-1204; GENEVIÈVE, Lessard. 'Preventive Reparations at a Crossroads: The Inter-American Court of Human Rights and Colombia's Search for Peace'. The International Journal of Human Rights, v. 22, n. 9, p. 1221, 2018. Como se mencionó anteriormente, Lessard defiende implicitamente un enfoque individual respecto a reparaciones por parte de la CtIDH en los casos examinados por lo menos respecto a indemnización. 
trará que el enfoque de caso individual en el tipo de casos examinados es problemático desde el punto de vista jurídico, analítico y normativo. Segundo, este artículo propondrá un enfoque alternativo para responder de manera satisfactoria a las dificultades jurídicas y normativas que plantea un enfoque de caso individual. Para fundamentar estas dos proposiciones, el presente artículo utilizará aportes analíticos y desarrollos recientes en la práctica internacional en derechos humanos y en la adjudicación comparada en materia de derechos fundamentales, particularmente respecto a formas generales de control judicial por parte de tribunales de derecho público. ${ }^{38}$ Tercero, este artículo tendrá en cuenta y complementará trabajos académicos existentes sobre temas más amplios sobre reparaciones en contextos de transición. Esos temas incluyen los efectos negativos que puede tener la significativa disparidad entre las medidas de compensación y rehabilitación ordenadas por la CtIDH y las proporcionadas por un PMR. ${ }^{39}$ También incluyen la cuestión de chasta qué punto es aplicable el principio de la reparación integral en contextos de transición ${ }^{40}$ En particular, además de examinar una cuestión no abordada en esta literatura, este artículo hace las siguientes contribuciones. En primer lugar, examina esos temas más amplios desde una perspectiva de derecho internacional y normativa, y su efecto agregado sobre el tema analizado. En segundo lugar, hace nuevos aportes basado en la jurisprudencia comparada en materia de derechos fundamentales y la práctica internacional de derechos humanos.

\footnotetext{
38 Ver por ejemplo, Langford, 'Social Rights Adjudication', 184, 192-304; Rodríguez-Garavito y Rodríguez-Franco, Juicio a La Exclusion; Tsereteli, 'The Role of the European Court of Human Rights in Facilitating Legislative Change in Cases of Long-Term Delays in Implementation.'

39 Mersky y Roht-Arriaza, 'Guatemala', 19-20, 29-30; Roht-Arriaza, 'Central America, the Inter-American System, and Accountability for International Crimes', UC Hastings Research Paper, 2012, no 6; Oficina del Alto Comisionado de las Naciones Unidas para los Derechos Humanos, 'Rule of Law Tools for Post-Conflict States: Reparations Programmes', 35-36. Para un análisis más general, ver Antkowiak, 'Remedial Approaches to Human Rights Violations'.

${ }^{40}$ Ver por ejemplo, Pablo de Greiff, 'Justice and Reparations'; Rashida Manjoo, Informe de la Relatora Especial sobre la Violencia contra la Mujer, sus causas y consecuencias, 23 abril 2010, doc. de la ONU A/ HRC/14/22, párrafo 18.
}

\section{Principales enfoques adoptados por la Corte Interamericana}

En esta sección se analizarán los principales enfoques adoptados por la CtIDH con respecto al alcance del control judicial que esta Corte debe ejercer en los casos estudiados. Particularmente, los enfoques de esta Corte a la pregunta: ¿qué tipo de ordenes de reparaciones debe adoptar esta Corte en los casos examinados? Para esto, se analizaron las decisiones adoptadas por la CtIDH entre junio de 2013 y noviembre de 2018. Los principales criterios utilizados para establecer este período fueron: Primero, la CtIDH ha emitido la mayoría de sus decisiones que abordan de manera sustantiva la cuestión analizada durante este período. Segundo, las investigaciones anteriores abarcan las decisiones dictadas hasta octubre de $2016 .{ }^{41}$

La CtIDH adoptó un enfoque de caso individual en sus órdenes de reparaciones en sus decisiones dictadas desde al menos noviembre de 2004 hasta octubre de 2013. De conformidad con ese enfoque, esta corte exigió la plena observancia del principio de la reparación integral. ${ }^{42}$ Este Tribunal no examinó los PMR durante este período. Por ejemplo, en el caso Masacres de Río Negro contra Guatemala, decidido en septiembre de 2012, la Corte ordenó que cualquier dinero pagado a las víctimas en el caso a través del PMR de Guatemala debía restarse de las reparaciones que ordenaría. ${ }^{43}$

En noviembre de 2013, la CtIDH cambió su posición sobre este tema. En el caso de Génesis contra Colombia, decidido en noviembre de 2013, la CtIDH abrió la puerta para ejercer control judicial sobre los PMR. La CtIDH definió varios requisitos sustantivos que estos

${ }^{41}$ Ver SANDOVAL, Clara. 'Two Steps Forward, One Step Back: Reflections on the Jurisprudential Turn of the Inter-American Court of Human Righs on Domestic Reparation Programmes'. The International Journal of Human Rights, 22, n. 9, 2018.

${ }^{42}$ Ver CTIDH. caso de la masacre de Plan de Sánchez contra Guatemala, sentencia de reparaciones, 19 de noviembre de 2004; CTIDH. caso masacres de Río Negro vs. Guatemala, sentencia de excepción preliminar, fondo, reparaciones y costas, 4 de Septiembre de 2012, párrafo 304. Para un análisis de los casos decididos durante ese periodo, ver Sandoval, 'Two Steps Forward, One Step Back'. Para un análsis del caso de Plan de Sánchez Massacre v. Guatemala, ver abajo en la próxima sección, la subsección "Un enfoque de caso individual respecto a reparaciones conduce involuntariamente a un trato diferente a víctimas en situaciones similares".

${ }^{43}$ CTIDH. caso masacres de Río Negro vs. Guatemala, sentencia de excepción preliminar, fondo, reparaciones y costas, 4 de Septiembre de 2012, párrafo 304. 
programas deben cumplir para que esta Corte pueda remitirse a lo que establece un determinado PMR en su decisión sobre reparaciones. En este caso, la CtIDH afirmó lo siguiente:

\begin{abstract}
En [escenarios de justicia transicional], [los programas administrativos de reparación] deben entenderse en conjunto con otras medidas de verdad y justicia, siempre y cuando se cumplan con una serie de requisitos relacionados ... con su legitimidad - en especial, a partir de la consulta y participación de las víctimas-; su adopción de buena fe; el nivel de inclusión social que permiten; la razonabilidad y proporcionalidad de las medidas pecuniarias ...

Asimismo, un criterio de justicia respecto a la reparación pecuniaria debe involucrar aspectos que, en el contexto específico, no resulten ilusorios o irrisorios y permitan una contribución real para que la víctima enfrente las consecuencias negativas que dejaron las violaciones de derechos humanos en su vida. $^{44}$
\end{abstract}

Sin embargo, la CtIDH no examinó si el PMR de Colombia establecido en la Ley de Víctimas cumplía con esos requisitos sustantivos. No obstante, la CtIDH mencionó que la Corte Constitucional de Colombia había reconocido que este PMR había sido un avance importante en este tema. ${ }^{45} \mathrm{La}$ CtIDH también destacó varias características de la legislación de Colombia que establecen medidas de asistencia, atención, reparación integral y restitución de tierras a las víctimas pertenecientes a las comunidades negras, afrocolombiana, raizal y palenquera. ${ }^{46}$ En este caso, la CtIDH se remitió a lo que establece el PMR de Colombia respecto a algunas formas de reparación, en particular indemnización y rehabilitación. ${ }^{47}$

Considero que el hecho de que la CtIDH no haya examinado si el PMR de Colombia cumplía los requisitos sustantivos que esta Corte estableció es motivo de gran preocupación. Esta evaluación es necesaria para asegurar que el control judicial de los PMR por parte de esta Corte garantice plenamente los derechos de las víctimas y el papel de supervisión de la Corte.

En Yarce y otros contra Colombia, la CtIDH reiteró los requisitos sustantivos que enumeró en Genesis. ${ }^{48} \mathrm{La}$ Cor-

\footnotetext{
44 CTIDH. Operación Génesis vs. Colombia, 20 de noviembre de 2013, párrafos 470-471.

${ }_{45}$ CTIDH. Operación Génesis vs. Colombia, 20 de noviembre de 2013, párrafo 472.

46 CTIDH. Operación Génesis vs. Colombia, 20 de noviembre de 2013, párrafo 473.

47 Ibid., párrafos 453, 475.

${ }^{48}$ CTIDH. caso de Yarce y otros vs. Colombia, sentencia 22 de noviem-
}

te agregó un requisito de procedimiento al afirmar lo siguiente:

[E]n relación con la solicitud del Estado de que en la presente sentencia se haga una remisión al programa administrativo de reparaciones [de Colombia], la Corte entiende que para tales efectos hubiera sido necesario que Colombia ... precise e individualice ... la forma en que [las medidas de reparación establecidas en ese programa] aplicarían a cada una de las víctimas del caso ..." ${ }^{\prime 9}$

Con base en este requisito de procedimiento, la CtIDH rechazó la solicitud de Colombia de que la Corte se remitiera al PMR de Colombia en su decisión sobre reparaciones. Una vez más, la Corte no analizó si el PMR de Colombia cumplía los requisitos sustantivos que identificó.

Sin embargo, en dos decisiones de la CtIDH dictadas entre noviembre de 2016 y noviembre de 2018, esta Corte exigió de nuevo el cumplimiento pleno con el principio de la reparación integral. Con base en este requisito, la Corte rechazó las solicitudes de remisiones a los PMR realizadas por los Estados involucrados en estos casos. En el caso de la Aldea de Chichupac y comunidades vecinas del municipio de Rabinal contra Guatemala, ${ }^{50}$ uno de los principales argumentos que la CtIDH utilizó para rechazar la solicitud de Guatemala de que la Corte se remitiera al PMR de Guatemala en su decisión sobre reparaciones en el caso fue: "el Estado no ha demostrado que haya proporcionado una reparación integral a las víctimas en el caso...." ${ }^{51}$ Basándose en este y otras razones, la Corte concluyó que procedería a evaluar la cuestión de las reparaciones en el caso de acuerdo con sus propios estándares y se centró en el caso individual. ${ }^{52}$

De igual manera, en su decisión en el caso vereda la Esperanza contra Colombia, dictada el 31 de agosto de 2017 y

bre de 2016, párrafo 326.

${ }^{49}$ CTIDH. caso de Yarce y otros vs. Colombia, sentencia 22 de noviembre de 2016, párrafo 328.

${ }^{50}$ CTIDH. caso de la Aldea de Chichupac y comunidades vecinas del municipio de Rabinal contra Guatemala, sentencia de excepción preliminar, fondo, reparaciones y costas, 30 de noviembre de 2016 (en adelante "caso de la Aldea de Chichupac contra Guatemala").

51 CTIDH. caso de la Aldea de Chichupac y comunidades vecinas del municipio de Rabinal contra Guatemala, sentencia de excepción preliminar, fondo, reparaciones y costas, 30 de noviembre de 2016 (en adelante "caso de la Aldea de Chichupac contra Guatemala"), párrafo 280.

${ }^{52}$ CTIDH. caso de la Aldea de Chichupac y comunidades vecinas del municipio de Rabinal contra Guatemala, sentencia de excepción preliminar, fondo, reparaciones y costas, 30 de noviembre de 2016 (en adelante "caso de la Aldea de Chichupac contra Guatemala"), párrafo 281. 
que es la más reciente decisión de la CtIDH sobre el tema estudiado hasta noviembre de 2018, esta Corte sostuvo que para decidir si debe remitirse a un determinado PMR en su decisión de reparaciones en un caso determinado:

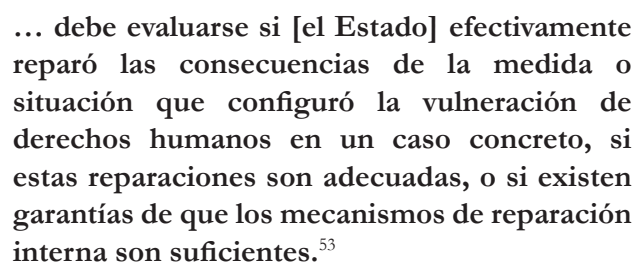

Vistas en conjunto, las tres alternativas del test jurídico adoptado por la CtIDH y la forma en que esta Corte ha interpretado estas alternativas en sus decisiones anteriores hacen razonable inferir que la Corte exige nuevamente la observancia del principio de la reparación integral como condición para remitirse a un PMR específico en un caso determinado. Sin embargo, en este caso, la Corte rechazó la solicitud de Colombia de remitirse al PMR de Colombia en su decisión sobre reparaciones en el caso, basándose en otros motivos. ${ }^{54}$ Por tanto, la Corte volvió a su posición inicial, basada en el principio de reparación integral.

En conclusión, en la mayoría de las decisiones dictadas entre junio de 2013 y noviembre de 2018, la CtIDH adoptó un enfoque de caso individual en sus órdenes de reparaciones en el tipo de casos analizados. El principio de la reparación integral fue el argumento fundamental que esta Corte usó para adoptar este enfoque. Cabe señalar que la CtIDH ha sido una firme defensora del principio de la reparación integral frente a violaciones de los derechos humanos, desde sus primeras decisiones en casos contenciosos. ${ }^{55} \mathrm{La}$ Corte ha hecho esto basándose en la jurisprudencia ${ }^{56}$ y la práctica internacional. ${ }^{57}$

\footnotetext{
53 CTIDH. caso de la Vereda La Esperanza vs. Colombia, 31 de agosto de 2017, párrafo 263.

${ }^{54}$ CTIDH. caso de la Vereda La Esperanza vs. Colombia, 31 de agosto de 2017, párrafos 264-265.

55 Ver por ejemplo, CTIDH. Velasquez Rodriguez vs. Honduras, sentencia de reparaciones, 21 de julio de 1989, párrafo 26; CTIDH. Juan Humberto Sánchez vs. Honduras, sentencia de 7 de junio de 2003, párrafo 149. Para análisis de este tema por parte de académicos, ver Sandoval, 'Two Steps Forward, One Step Back'; Antkowiak, 'Remedial Approaches to Human Rights Violations'.

${ }^{56}$ Ver por ejemplo Tribunal Permanente de Justicia Internacional, Factory at Chorzón, Alemania c. Polonia, reclamación por indemnización, sentencia No 13, (1928) PCIJ Series A No 17, ICGJ 255 (PCIJ 1928), 13 septiembre 1928, párrafo 47; Corte Internacional de Justicia, LaGrand (Alemania c. Los Estados Unidos), fondo, sentencia de 27 de junio de 2001, párrafo 48. Ver CTIDH. Velasquez Rodriguez vs. Honduras, sentencia de reparaciones, 21 de julio de 1989, párrafos 25-28.

57 Ver por ejemplo, el artículo 31 y su comentario en el proyecto de artículos sobre la responsabilidad del Estado de 2001 preparado por
}

\section{Un enfoque de caso individual: un análisis crítico}

En esta sección, se analizará de manera crítica el enfoque de caso individual respecto a órdenes de reparaciones adoptado por la CtIDH en el tipo de casos estudiados. Se demostrará que hay varios defectos en este enfoque desde una perspectiva jurídica, analítica y normativa.

\subsection{Diferenciando entre acceso y adjudicación definida de manera estricta ante la CtIDH, y la aplicación estricta del principio de la reparación integral}

Primero, debe diferenciarse entre acceso y adjudicación definida de manera estricta ante la CtIDH, por un lado, y la aplicación estricta del principio de la reparación integral, por el otro lado. Estas se refieren a cuestiones diferentes. La garantía de acceso y de adjudicación estrictamente definida ante la CtIDH se refieren a acceso y a adjudicación estrictamente definida. Por el contrario, la aplicación estricta del principio de la reparación integral se refiere a las medidas de reparación. Debe especificarse que, en este contexto, 'aplicación estricta’ significa que el principio de la reparación integral debe ser aplicado independientemente de las características del PMR implementado en el Estado demandado.

La práctica constitucional comparada e investigaciones sólidas sobre derechos fundamentales confirman que esas categorías se refieren a cuestiones diferentes. Según Malcolm Langford, la adjudicación de derechos suele implicar cuatro elementos: "1) la identificación de las obligaciones pertinentes; 2) la selección de [juicios o test jurídicos]; 3) la aplicación de dichas obligaciones a la reclamación a la luz de los hechos relevantes; y 4) dictar las ordenes de reparaciones según sea necesario". ${ }^{58}$ Los tres primeros elementos se refieren a la "adjudicación estrictamente entendida", mientras que el cuarto se refiere a diseñar y ordenar las medidas de reparación. Otros académicos reconocidos también han hecho la

la Comisión de Derecho Internacional de las Naciones Unidas en CRAWFORD, James. (ed.) The International Law Commission's Articles on State Responsibility: Introduction, Text, and Commentaries. Cambridge: Cambridge University Press, 2002. p. 201 et seq.

${ }^{58}$ LANGFORD, Malcolm. 'Social Rights Adjudication. An Interdisciplinary Perspective'. Tesis (Doctorado) Facultad de Derecho, Universidad de Oslo, 2014. p. 206-207. 
distinción entre "adjudicación estrictamente entendida" y diseñar y ordenar las medidas de reparación. ${ }^{59}$ Con respecto a las dos últimas, la práctica constitucional comparada demuestra que las cortes de derecho público pueden ordenar muchos tipos de reparaciones más allá de la aplicación estricta del principio de la reparación integral en el caso individual involucrado. Estas órdenes de reparación comprenden control de leyes y declaraciones de invalidez de las mismas con efecto hacia el futuro. ${ }^{60}$

Igualmente, es razonable argumentar que el derecho a la garantía y a la protección judicial reconocido en la Convención Americana de Derechos Humanos abarca acceso y adjudicación estrictamente definida ante la CtI$\mathrm{DH}$. Por el contrario, este derecho no requiere necesariamente la aplicación estricta del principio de la reparación integral en el tipo de casos examinados.

\section{2 ¿Debe aplicarse el principio de la reparación integral en contextos de transición?}

Como se analizó anteriormente, el principio de la reparación integral ha sido considerado por la CtIDH y varios académicos como un fundamento clave del enfoque de caso individual en las decisiones de reparaciones de la CtIDH. Por consiguiente, surge la pregunta si el principio de la reparación integral debe informar las medidas de reparaciones adoptadas en contextos de transición, incluyendo aquellas adoptadas por la CtIDH.

Como se mencionó previamente, un número significativo de expertos internacionales en derechos humanos ha criticado la aplicación del principio de la reparación integral en contextos de transición. Primero, estos expertos han sostenido que este principio se elaboró partiendo del supuesto de que lo que se pretende es la resolución de casos relativamente aislados. ${ }^{61}$ Por el con-

59 Ver por ejemplo, Rodríguez-Garavito y Rodríguez-Franco, Juicio a La Exclusión; Mark Tushnet, Weak Courts, Strong Rights: Judicial Review and Social Welfare Rights in Comparative Constitutional Law (Princeton: Princeton University Press, 2009). Tsereteli hace un diferenciación similar. Ver Nino Tsereteli, 'Emerging doctrine of deference of the Inter-American Court of Human Rights?', The International Journal of Human Rights, 20:8 (2016), 1097-1112.

${ }^{60}$ Ver por ejemplo, ROACH, Kent. 'The Challenges of Crafting Remedies for Violations of Socio-Economic Rights', en: LANGFORD, Malcolm. (ed.). Social Rights Jurisprudence: Emerging Trends in International and Comparative Law. Cambridge: Cambridge University Press, 2009.

${ }^{61}$ Ver por ejemplo, DE GREIFF, Pablo. 'Justice and Reparations', en The Handbook of Reparations. Oxford: Oxford University Press, trario, los contextos de transición suelen involucrar violaciones de los derechos humanos en gran escala y, por lo tanto, un gran número de víctimas. Por ejemplo, según el Registro Nacional de Víctimas de Colombia, hay más de ocho millones de víctimas del conflicto armado interno en Colombia. ${ }^{62}$ Esta es una de las principales razones que Pablo de Greiff dio a favor de establecer e implementar PMR en contextos de transición. ${ }^{63}$ En su informe de 2010 como Relatora Especial de la ONU sobre la Violencia contra la Mujer, Rashida Manjoo también criticó la aplicación del principio de la reparación integral en contextos de transición:

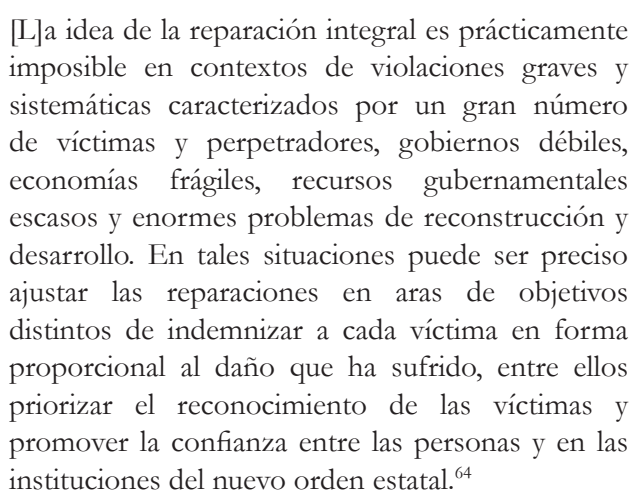

Incluso la CtIDH ha reconocido en algunas decisiones que en contextos de justicia transicional el cumplimiento del principio de la reparación integral no debe ser la única forma de satisfacer el derecho a la reparación de las víctimas de violaciones de los derechos humanos. Según esta corte, en dichos contextos los PMR son otra forma legítima de satisfacer ese derecho. Por ejemplo, en Génesis contra Colombia, ${ }^{65}$ la CtIDH declaró lo siguiente:

[L]a Corte resalta que el Derecho Internacional contempla la titularidad individual del derecho a la reparación. Sin perjuicio de ello, el Tribunal indica que, en escenarios de justicia transicional en los cuales los Estados deben asumir su deber de reparar masivamente a números de víctimas que exceden ampliamente las capacidades y posibilidades de los

\section{6.}

62 Según el Registro Nacional de Víctimas de Colombia, al 31 de diciembre de 2018 había 8.405.614 víctimas del conflicto armado interno en Colombia, de las cuales 6.823.224 tienen derecho a atención y reparaciones a través del PMR. Ver https://www.unidadvictimas.gov.co/es/registro-unico-de-victimas-ruv/37394

63 Ver por ejemplo, DE GREIFF, Pablo. 'Justice and Reparations', en The Handbook of Reparations. Oxford: Oxford University Press, 2006. p. 454-455.

64 Rashida Manjoo, Informe de la Relatora Especial sobre la Violencia contra la Mujer, sus causas y consecuencias, 23 abril 2010, doc. de la ONU A/ HRC/14/22, párrafo 18.

65 CTIDH. Operación Génesis vs. Colombia, 20 de noviembre de 2013. 
tribunales nacionales, los programas administrativos de reparación constituyen una de las maneras legítimas de satisfacer el derecho a la reparación. ${ }^{66}$

Como puede verse, la CtIDH destacó el gran número de violaciones de los derechos humanos y de víctimas en contextos de transición, y la limitada capacidad de los tribunales nacionales. Varios Estados demandados en casos ante la CtIDH han presentado argumentos similares a los anteriores. ${ }^{67}$

Estas características particulares de los contextos de transición fueron los factores que dieron lugar a la justicia transicional. Como explica el Centro Internacional para la Justicia Transicional, la justicia transicional es "una justicia adaptada a sociedades que se transforman a si mismas después de un período de violación generalizada de los derechos humanos". ${ }^{68}$ En la mayoría de los casos, en este tipo de escenario, esto ha significado adoptar estándares más bajos que los aplicados a violaciones aisladas de los derechos humanos. Por lo tanto, es razonable sostener que, si las condiciones de un determinado contexto de transición lo exigen, debe ajustarse el estándar de la reparación integral. Por ejemplo, se podrían establecer niveles mínimos de cumplimiento, en particular en lo que respecta a la indemnización y la rehabilitación..$^{69}$ El caso de la Masacre de Plan de Sánchez. contra Guatemala, el cual se examinará en la próxima sección, ilustra este punto.

El análisis precedente demuestra que la aplicación del principio de la reparación integral a todas las violaciones de los derechos humanos perpetradas en las situaciones analizadas impondría por lo general una carga financiera insoportable o afectaría desproporcionadamente la capacidad del Estado en transición para cumplir otros objetivos legítimos. Este sería el caso par-

${ }^{66}$ CTIDH. Operación Génesis vs. Colombia, 20 de noviembre de 2013, párrafo 470 .

${ }^{67}$ See e.g., Gobierno de Guatemala, Presidencia de la Republica, Comisión Presidencial Coordinadora de la Política del Ejecutivo en Materia de Derechos Humanos COPEDEH, Alegatos Finales del Estado de Guatemala dentro del Caso 12.599 Florencio Chitay v. Guatemala, Presentado ante la Honorable Corte Interamericana de Derechos Humanos, 3 de marzo de 2010, 17 y 18; Gobierno de Colombia, Alegatos Finales Escritos, República de Colombia, Caso No. 12.573 "Marino López Mena Y Otros- Operación Génesis", 142-144, 152.

${ }^{68}$ Centro Internacional para la Justicia Transicional, 'Qué es la justicia transicional?', en Fichas ed. Centro International para la Justicia Transicional (Nueva York, 2009).

${ }^{69}$ Ver por ejemplo, BOLIVAR J., Aura Patricia. Programas Administrativos de Reparación: El Caso Colombiano en Perspectiva' Tesis (Magister en derecho) Universidad Nacional de Colombia, 2012. ticularmente respecto a medidas de indemnización y de rehabilitación. Por consiguiente, sería excesivo o desproporcionado en términos de derecho internacional exigir el cumplimiento de este principio, particularmente respecto a las dos formas de reparación mencionadas, en tales circunstancias.

\subsection{Un enfoque de caso individual por parte de la Corte Interamericana conduce involuntariamente a un trato diferente a víctimas en situaciones similares}

Como se mencionó anteriormente, las reparaciones individuales ordenadas por tribunales tradicionalmente se basan en el principio de la reparación integral. Las concedidas por PMR no. Entonces, un enfoque de caso individual por parte de la CtIDH conduce involuntariamente a un tratamiento diferente de víctimas en situaciones similares. Por ejemplo, Mersky y Roht-Arriaza documentaron varios problemas que surgieron en Guatemala debido a la disparidad significativa entre las medidas de indemnización y rehabilitación ordenadas por la CtIDH en los casos presentados ante esta Corte, y las medidas establecidas por el Programa Nacional de Reparaciones. ${ }^{70}$ Por lo tanto, un enfoque de caso individual respecto a las órdenes de reparaciones por parte de la CtIDH conduce involuntariamente a una situación que va en contra del principio de equidad. ${ }^{71}$ Esto es particularmente problemático debido a las mencionadas características de los contextos en transición. Además, esas disparidades pueden afectar negativamente la percepción general de las víctimas sobre la justicia y la equidad de un determinado PMR. ${ }^{72}$

El caso de la Masacre de Plan de Sánchez contra Guatemala, mencionado previamente, ilustra las deficiencias que tiene un enfoque de caso individual por parte de la CtI$\mathrm{DH}$ a la hora de decidir casos que tienen lugar en situa-

\footnotetext{
${ }^{70}$ Mersky y Roht-Arriaza, 'Guatemala', 19-20, 29-30. Ver también Roht-Arriaza, 'Central America, the Inter-American System, and Accountability for International Crimes'.

${ }^{71}$ Respecto a esta preocupación en relación con reparaciones por violaciones de derechos humanos a gran escala en general por la CTIDH. ver Jo M. Pasqualucci, 'Victim Reparations in the InterAmerican Human Rights System: A Critical Assessment of Current Practice and Procedure', Michigan Journal of International Law 18 (1996).

72 Oficina del Alto Comisionado de las Naciones Unidas para los Derechos Humanos, 'Rule of Law Tools for Post-Conflict States: Reparations Programmes', 35-36. Para un análisis más general, ver Antkowiak, 'Remedial Approaches to Human Rights Violations'.
} 
ciones de violaciones masivas de los derechos humanos. La CtIDH determinó que se violaron los derechos a la vida, al trato humano, a la propiedad y a la protección judicial, entre otros derechos. ${ }^{73}$ Guatemala solicitó a la Corte que se remitiera a su PMR en la decisión de la Corte sobre reparaciones. ${ }^{74} \mathrm{La} \mathrm{CtIDH}$ no abordó los argumentos de Guatemala y no proporcionó ninguna razón jurídica para explicar esta posición. Las indemnizaciones ordenadas por la CtIDH fueron en total 7,9 millones de dólares. ${ }^{75}$ Esto. incluía 5.000 dólares a cada víctima como compensación por daños pecuniarios, ${ }^{76}$ y 20.000 dólares a cada víctima como compensación por daños no pecuniarios. ${ }^{77} \mathrm{La}$ Corte también ordenó a Guatemala "poner en marcha un programa de vivienda para proporcionar una vivienda adecuada ${ }^{78}$ y ofrecer tratamiento de salud a las víctimas sobrevivientes". ${ }^{79}$

El enfoque de caso individual respecto a las órdenes de reparaciones adoptado por la CtIDH en este caso es preocupante. Primero, como se puede observar, las reparaciones ordenadas por la CtIDH implicaron importantes recursos financieros. Segundo, como se mencionó, anteriormente, este caso fue, según la Comisión de la Verdad de Guatemala, sólo una de las 626 masacres perpetradas por miembros de la Fuerza Pública durante el conflicto armado en Guatemala. ${ }^{80}$ Por último, hubo una disparidad significativa entre las medidas de indemnización y rehabilitación ordenadas por la CtIDH y las concedidas por el PMR de Guatemala. Por lo tanto, en aquellas situaciones en las que se ha establecido un PMR, sería más apropiado que la CtIDH evaluara a fondo el PMR respectivo. Esto dada las características de los escenarios de transición y el principio de equidad.

\footnotetext{
73 CTIDH. caso de la masacre de Plan de Sánchez contra Guatemala, sentencia de fondo, 29 de abril de 2004, párrafos 47, 52.

74 Presidencia de la Republica de Guatemala, COPREDEH, 21 de mayo de 2004, RED-A-28-2004, punto 7, 15.

75 MERSKY, Marcie; ROHT-ARRIAZA, Naomi. 'Guatemala'. En: SALAZAR, Katya; ANTKOWIAK,Thomas. (ed.). Victims Unsilenced: The Inter-American Human Rights System and Transitional Justice in Latin America. Washington, DC: Due Process of Law Foundation, 2007.

${ }^{76}$ CTIDH. caso de la masacre de Plan de Sánchez contra Guatemala, sentencia de reparaciones, 19 de noviembre de 2004, párrafo 74.

${ }^{77}$ CTIDH. caso de la masacre de Plan de Sánchez contra Guatemala, sentencia de reparaciones, 19 de noviembre de 2004, párrafo 107.

78 CTIDH. caso de la masacre de Plan de Sánchez contra Guatemala, sentencia de reparaciones, 19 de noviembre de 2004, párrafo 105.

${ }^{79}$ CTIDH. caso de la masacre de Plan de Sánchez contra Guatemala, sentencia de reparaciones, 19 de noviembre de 2004, párrafo 107.

${ }^{80}$ Gobierno de Guatemala, Comisión para el Esclarecimiento Histórico (1999). Guatemala: Memoria del Silencio, Conclusiones y Recomendaciones, párrafo 86.
}

Como se mencionó, por definición los PMR tienen un alcance general.

Varios Estados demandados ante la CtIDH han presentado argumentos similares a los presentados en esta sección. ${ }^{81}$ Las investigaciones existentes sobre el tema estudiado no han abordado este efecto involuntario de un enfoque de caso individual en los casos estudiados. ${ }^{82}$

\subsection{Limitarse a un enfoque de caso individual en los casos estudiados es injustificado teniendo en cuenta los poderes de la Corte Interamericana}

El punto de vista según el cual la CtIDH debe limitarse a un enfoque individual en sus órdenes de reparación en los casos estudiados es injustificado teniendo en cuenta los poderes de esta corte. Esos poderes deben ser interpretados teniendo en cuenta la práctica internacional en derechos humanos, la jurisprudencia de la propia CtIDH y la práctica constitucional comparada.

En primer lugar, en la adjudicación comparada de derechos fundamentales se reconoce ampliamente que los tribunales de derecho público pueden, por lo general, ejercer formas generales de control judicial. Como se mencionó anteriormente, en formas generales de control judicial un tribunal examina un patrón de violaciones de los derechos humanos o abusos repetitivos causados por factores comunes, y utiliza procedimientos que tienen por objeto adoptar medidas generales para abordar esos factores. ${ }^{83}$ Los tribunales de derecho público de varios países y varios órganos internacionales han utilizado formas generales de control judicial para abordar reclamaciones de violaciones sistemáticas y repetitivas a los derechos humanos. ${ }^{84} \mathrm{El}$ procedimiento

${ }^{81}$ See e.g., Gobierno de Guatemala, Presidencia de la Republica, Comisión Presidencial Coordinadora de la Política del Ejecutivo en Materia de Derechos Humanos COPEDEH, Alegatos Finales del Estado de Guatemala dentro del Caso 12.599 Florencio Chitay v. Guatemala, Presentado ante la Honorable Corte Interamericana de Derechos Humanos, 3 de marzo de 2010, 17 y 18.

82 SANDOVAL, Clara. 'Two Steps Forward, One Step Back: Reflections on the Jurisprudential Turn of the Inter-American Court of Human Righs on Domestic Reparation Programmes'. The International Journal of Human Rights, 22, n. 9, 2018.

83 Ver por ejemplo, Langford, 'Social Rights Adjudication', 184, 192304; Rodríguez-Garavito y Rodríguez-Franco, Juicio a La Exclusión.

${ }^{84}$ Respecto a esta forma de control judicial en relación con violaciones a los derechos económicos y sociales, ver por ejemplo, Langford, 'Social Rights Adjudication', 184, 192-304; BILCHITZ, David. 'Constitutionalism, the Global South, and Economic Justice', en Constitutionalism of the Global South: The Activist Tribunals of India, 
de sentencia piloto del Tribunal Europeo de Derechos Humanos es un buen ejemplo de una forma general de control judicial de derechos civiles por un tribunal regional de derechos humanos. ${ }^{85}$

En segundo lugar, la CtIDH ha ejercido de manera consistente formas generales de control judicial, en particular en sus órdenes de reparaciones. ${ }^{86}$ Cabe señalar que, en el tipo de casos estudiados, la CtIDH ejercería una forma general de control judicial relativamente limitada. En primer lugar, existiría una política pública de reparaciones, es decir, un PMR, en el país en cuestión. En segundo lugar, como se examinará en la siguiente sección, es probable que el control por parte de la CtI$\mathrm{DH}$ de algunos aspectos de un PMR específico sea de baja intensidad.

Tercero, el artículo 63, párrafo 1, de la Convención Americana de Derechos Humanos, que establece las facultades de la CtIDH para ordenar reparaciones, es

South Africa, and Colombia, ed. Daniel Bonilla Maldonado. Cambridge: Cambridge University Press, 2013; RODRÍGUEZ-GARAVITO, César; RODRÍGUEZ-FRANCO, Diana. Juicio a La Exclusión: El Impacto de los Tribunales sobre los Derechos Sociales en el Sur Global. Buenos Aires: Siglo 21 Editora Iberoamericana, 2015.

85 Para análisis de este tema por parte de académicos, ver por ejemplo, TSERETELI, Nino. 'The Role of the European Court of Human Rights in Facilitating Legislative Change in Cases of LongTerm Delays in Implementation'. En: FØLLESDAL, Andreas; ULFSTEIN, Geir; SAUL, Matthew. (ed.). The International Human Rights Judiciary and National Parliaments: Europe and Beyond, Studies on Human Rights Conventions. Cambridge: Cambridge University Press, 2017.

86 Para análisis de este tema por parte de académicos, ver por ejemplo, HUNEEUS, Alexandra. 'Reforming the State from Afar: Structural Reform Litigation at the Human Rights Courts'. Yale Journal of International Law, v. 40, n. 1, p. 1-40, 2015, LONDOÑO LÁZARO, María Carmelina; HURTADO, Mónica. 'Las Garantías De No Repetición en la Práctica Judicial Interamericana y su Potencial Impacto en la Creación del Derecho Nacional'. Boletin mexicano de derecho comparado, 50, 2017; CORNEJO CHAVEZ, Leiry. 'New Remedial Responses in the Practice of Regional Human Rights Courts: Purposes Beyond Compensation'. International Journal of Constitutional Law, v. 15, n. 2, 2017; GUARNIZO, Diana. 'Guarantees of NonRepetition and the Right to Health: Review of the Law and Evolving Practice of Judicial and Semi-Judicial Bodies at Global and Regional Levels'. Tesis (Doctorado) University of Essex, 2106. p. 156-180; ATTANASIO, David L. 'Extraordinary Reparations, Legitimacy, and the Inter-American Court. University of Pennsylvania Journal of International Law, v. 37, n. 3, p. 813-71, 2016; RUBIO-MARÍN Ruth; SANDOVAL, Clara. 'Engendering the Reparations Jurisprudence of the Inter-American Court of Human Rights: The Promise of the 'Cotton Field' Judgment'. Human Rights Quarterly, 33, n. 4, 2011; Sofía Galván, 'Legislative measures as guarantees of non-repetition: a reality in the InterAmerican Court, and a possible solution for the European Court', Revista IIDH 49, (2009); Antkowiak, 'Remedial Approaches to Human Rights Violations'. general. ${ }^{87}$ Esta disposición sugiere que la $\mathrm{CtIDH}$ tiene cierta discreción para diseñar y ordenar medidas de reparación. Además, la facultad de la CtIDH de evaluar PMR no ha sido impugnada en los casos en que esta Corte ha mantenido que tiene esa facultad (por ejemplo, el caso García Lucero y otros contra Chile). ${ }^{88}$

Por último, la práctica constitucional comparada e investigaciones sólidas sobre adjudicación de derechos fundamentales demuestran que los tribunales de derecho público pueden ordenar muchos recursos más allá de la aplicación estricta del principio de la reparación integral en relación con las personas involucradas en un caso individual.

En términos más generales, la posición según la cual la CtIDH debe examinar los PMR aplicables a casos individuales ante esta corte toma en consideración las principales dimensiones involucradas en la cuestión analizada mejor que otras posiciones que han sido sugeridas. Estas dimensiones incluyen: en primer lugar, las condiciones que suelen prevalecer en los contextos de transición - por ejemplo, el número significativo de víctimas involucradas y los recursos limitados de que disponen los Estados miembros para satisfacer sus numerosas necesidades. En segundo lugar, esta posición preservaría el rol de supervisión de la CtIDH en materia de reparaciones y, lo que es más importante, los PMR se someterían a control judicial por parte de la CtIDH. También garantizaría otros objetivos del SIDH, como la protección efectiva de los derechos humanos, ${ }^{89}$ el cual es razonable afirmar que se aplica a todas las víctimas en la misma situación en un país determinado. El enfoque propuesto garantizaría la coherencia de las reparaciones concedidas y el trato justo de las víctimas de violaciones de los derechos humanos en la misma situación en los países involucrados. Esto sería muy importante debido a las condiciones que suelen prevalecer en los entornos

\footnotetext{
87 Este provee lo siguiente: "1. Cuando decida que hubo violación de un derecho o libertad protegidos en esta Convención, la Corte dispondrá que se garantice al lesionado en el goce de su derecho o libertad conculcados. Dispondrá asimismo, si ello fuera procedente, que se reparen las consecuencias de la medida o situación que ha configurado la vulneración de esos derechos y el pago de una justa indemnización a la parte lesionada."

${ }^{88}$ CTIDH. caso García Lucero y otros vs. Chile, sentencia de 28 de Agosto de 2013, párrafo 189. Ver también CTIDH. caso de Manuel Cepeda Vargas vs. Colombia, sentencia de 26 de mayo de 2010, párrafo 246.

89 Ver por ejemplo, CTIDH. el Derecho a la Información sobre la Asistencia Consular, Opinión Consultiva OC-16/99 de 1 de octubre de 1999, Serie A No. 16, párrafo 58.
} 
de transición, en particular el número considerable de víctimas involucradas.

\section{5 ¿Es decisivo el principio de subsidiariedad?}

Algunos autores que han escrito sobre este tema parecen concebir el principio de subsidiariedad como absoluto y decisivo en el sentido de que conduciría necesariamente a uno de los extremos del espectro. ${ }^{90} \mathrm{El}$ primer extremo es que la CtIDH debería remitirse incondicionalmente a un determinado PMR en su decisión sobre reparaciones. El segundo extremo es que la CtIDH debe aplicar estrictamente el principio de la plena reparación. Esta subsección demuestra que el principio de subsidiariedad no es absoluto, sino que se aplica bajo ciertas condiciones. Por lo tanto, en este artículo se sostiene que este principio no es decisivo como se definió anteriormente. Como cuestión preliminar, esta subsección definirá el principio de subsidiariedad.

El principio de subsidiariedad proporciona un marco para la asignación de poder en aquellas situaciones en donde coexisten las funciones de órganos nacionales e internacionales. ${ }^{91}$ Jorge Contesse ha descrito la noción de subsidiariedad en el derecho internacional de la siguiente manera: Los Estados deben tener "prioridad prima facie" al decidir cuestiones cubiertas por instrumentos de derecho internacional. ${ }^{92}$ En el contexto del SIDH, el principio de subsidiariedad se puede describir de la siguiente manera:

El SIDH puede actuar en dos escenarios: En primer lugar, si el sistema nacional no está dispuesto o no puede investigar, juzgar o proporcionar reparaciones por las violaciones de los derechos humanos perpetradas en

\footnotetext{
90 Ver por ejemplo, GENEVIÈVE, Lessard. 'Preventive Reparations at a Crossroads: The Inter-American Court of Human Rights and Colombia's Search for Peace'. The International Journal of Human Rights, v. 22, n. 9, 2018. Sandoval también adopta implícitamente este enfoque en algunos de sus argumentos relacionados con el principio de subsidiariedad. Ver SANDOVAL, Clara. 'Two Steps Forward, One Step Back: Reflections on the Jurisprudential Turn of the Inter-American Court of Human Righs on Domestic Reparation Programmes'. The International Journal of Human Rights, 22, n. 9, 2018. 91 Ver por ejemplo, JACHTENFUCHS, Markus; KRISCH, Nico. 'Subsidiarity in Global Governance', 79 Law and Contemporary Problems, v. 79, 2016; CONTESSE, Jorge. 'Contestation and Deference in the Inter-American Human Rights System'. Law and Contemporary Problems, v. 79, p. 125, 2016.

${ }^{92}$ CONTESSE, Jorge. 'Contestation and Deference in the InterAmerican Human Rights System'. Law and Contemporary Problems, v. 79 , p. 125,2016
}

un Estado miembro. En segundo lugar, si un Estado miembro no ejerce la debida diligencia en la prevención de una de esas violaciones. ${ }^{93}$

Debe destacarse que el principio de subsidiariedad no es absoluto en el sentido de que deba aplicarse incondicionalmente. Como la CtIDH y varios académicos han reconocido, este se aplica bajo ciertas condiciones. ${ }^{94}$ Estas condiciones no están claramente definidas en la Convención Americana de Derechos Humanos ni en ningún otro instrumento del SIDH. Por lo tanto, la CtIDH tiene la tarea fundamental de establecer las condiciones en las que se aplican este principio. El enfoque propuesto, el cual se describe en la sección siguiente, incluye muchos de esas condiciones y, por tanto, podría ayudar a la Corte a decidir razonablemente sobre la aplicación de este principio en casos concretos.

Con base en todos los argumentos expuestos en esta sección, el punto de vista según el cual la CtIDH debe limitarse a un enfoque de caso individual en sus órdenes de reparaciones en el tipo de casos examinados es injustificado desde una perspectiva jurídica, analítica y normativa. Por lo tanto, la CtIDH debe adoptar un enfoque más matizado respecto a sus órdenes de reparaciones en el tipo de casos examinados.

\section{Un Enfoque Alternativo: Evaluación y Remisión Condicionada a los PMR}

En esta sección se expone el enfoque que propongo cuando la CtIDH deba resolver un caso que involucra a un Estado en el que se ha implementado un PMR. Este consiste en dos pasos: Primero, la CtIDH debe evaluar si un PMR específico cumple con las obligaciones del Estado en virtud de la Convención Americana de Derechos Humanos. En segundo lugar, la CtIDH debe examinar si se cumplen los requisitos adicionales para remitirse a un PMR específico en su decisión sobre reparaciones. En esta sección se abordarán las

\footnotetext{
93 Ver de manera general CTIDH. Velásquez Rodriguez contra Honduras, sentencia de fondo, 29 de julio de 1988, párrafo 166; y sentencia de reparaciones, 21 de julio de 1989.

${ }^{94}$ Ver por ejemplo, CTIDH. caso de Tenorio Roca y otros vs. Peru, sentencia de 22 de junio de 2016, párrafo 279. Para puntos de vista sobre este tema por parte de académicos, ver por ejemplo CAROZZA, Paolo. 'Subsidiarity as a Structural Principle of International Human Rights Law'. American Journal of International Law, v. 97, p. 78-79, 2003.
} 
siguientes cuestiones: En primer lugar, ¿qué criterios generales debe utilizar la CtIDH para evaluar si un determinado PMR cumple con las obligaciones del Estado en virtud de la Convención Americana de Derechos Humanos? En segundo lugar, ¿qué criterios adicionales debe utilizar la CtIDH para evaluar si un determinado PMR cumple con las obligaciones del Estado en virtud de la Convención Americana de Derechos Humanos? Por último, examinaré dos requisitos adicionales que la CtIDH debe evaluar para decidir si debe remitirse a un PMR específico en su decisión de reparaciones en un caso determinado. Cabe señalar que, como se analizó en la sección anterior, existen numerosas bases para la proposición de que la CtIDH puede, de acuerdo con sus funciones y facultades actuales, examinar la conformidad de los PMR aplicables a un caso ante esta Corte con las obligaciones de los Estados en virtud de la Convención Americana sobre Derechos Humanos.

\subsection{Criterios generales para evaluar si un determinado PMR cumple con las obligaciones de los Estados en virtud de la Convención Americana}

El primer tipo de criterios que la CtIDH debe aplicar para evaluar si un determinado PMR cumple con las obligaciones del Estado en virtud de la Convención Americana sobre Derechos Humanos son aquellos criterios generales aplicables a PMR en su conjunto. Estos abarcan elementos relativos a la adopción, el contenido y la implementación de un determinado PMR. Enseguida analizaré cada una de estas subcategorías.

\section{Criterios generales relacionados con aspectos sustantivos de los PMR}

Los requisitos generales relacionados con aspectos sustantivos de los PMR incluyen, en primer lugar, que las indemnizaciones individuales previstas en estos programas deben ir acompañadas del reconocimiento de responsabilidad y otras medidas para buscar verdad, justicia y garantías de no repetición..$^{5}$ Por lo tanto, la

\footnotetext{
95 Ver por ejemplo, CTIDH. Operación Génesis vs. Colombia, sentencia de 20 de noviembre de 2013, párrafo 470; CTIDH. caso de Yarce y otros vs. Colombia, sentencia de 22 de noviembre de 2016, párrafo 326; informe de DE GREIFF, Pablo. Informe del Relator Especial sobre la promoción de la verdad, la justica, la reparación y las garantías de no repetición, 14 de octubre de 2014, doc. de la ONU A/69/518, párrafos 11, 83. Para puntos de vista sobre este tema por parte de académicos, ver Moffett, 'Transitional Justice and Reparations'; Borneman, Political Crime and the Memory of Loss, 11.
}

CtIDH debe examinar la relación entre un PMR específico y otras medidas de justicia transicional, incluida la forma en que el conjunto de medidas incluidas en un PMR específico encaja con esas otras medidas. ${ }^{96} \mathrm{La}$ CtIDH debe aplicar un estándar estricto de control judicial al hacer esto, en particular respecto a aspectos directamente relacionados con los deberes del Estado de investigar, enjuiciar y sancionar violaciones graves de los derechos humanos. Los estándares de derecho internacional sobre estas obligaciones están bien establecidos. ${ }^{97}$

Según la jurisprudencia de la CtIDH, otros requisitos generales sobre aspectos sustantivos que los PMR deben cumplir incluyen tener en cuenta aspectos de género y permitir un cierto grado de inclusión social. ${ }^{98} \mathrm{El}$ Comité para la Eliminación de la Discriminación contra la Mujer y otros órganos internacionales también han dejado claro que reparaciones en contextos en transición deben tener en cuenta aspectos de género en su accesibilidad y en su contenido. ${ }^{99}$

\section{Requisitos generales relativos al proceso de di- seño, adopción e implementación de PMR}

El requisito general más importante relacionado con el proceso de diseño y adopción de un PMR es que el Estado en cuestión adopte un enfoque basado en los derechos humanos en dicho proceso. ${ }^{100}$ Esto incluye el proceso de toma de decisión sobre quién tiene derecho a reparaciones en virtud de un PMR y las violaciones de

${ }^{96}$ Ver por ejemplo, informe de DE GREIFF, Pablo. Informe del Relator Especial sobre la promoción de la verdad, la justica, la reparación y las garantías de no repetición, 14 de octubre de 2014, doc. de la ONU A/69/518, párrafos 11, 83.

97 Ver por ejemplo, OCHOA-SÁNCHEZ, Juan Carlos. The Rights of Victims in Criminal Justice Proceedings for Serious Human Rights Violations. Leiden: Martinus Nijhoff, 2013.

98 Ver por ejemplo, CTIDH. Operación Génesis vs. Colombia, sentencia de 20 de noviembre de 2013, párrafo 470; CTIDH. caso de Yarce y otros vs. Colombia, sentencia de 22 de noviembre de 2016, párrafo 326.

99 CEDAW, Recomendación General No. 35 sobre violencia de género contra las mujeres, 26 de julio de 2017, CEDAW/C/GC/35, párrafo 33. Ver también informe de DE GREIFF, Pablo. Informe del Relator Especial sobre la promoción de la verdad, la justica, la reparación y las garantías de no repetición, 14 de octubre de 2014, doc. de la ONU A/69/518; Diana Contreras Garduno, 'Collective Reparations: Tensions and Dilemmas between Collective Reparations with the Individual Right to Receive Reparations' (tesis de doctorado, Utrecht University, 2018).

100 Ver por ejemplo, informe de DE GREIFF, Pablo. Informe del Relator Especial sobre la promoción de la verdad, la justica, la reparación y las garantías de no repetición, 14 de octubre de 2014, doc. de la ONU A/69/518, párrafo 67; Moffett, 'Transitional Justice and Reparations', 33. 
los derechos humanos que el mismo abarcará. En lo que respecta a lo primero, la violación de los derechos humanos, independientemente de otras consideraciones, debe ser el requisito para dar acceso a los beneficios establecidos en un PMR. ${ }^{101}$ Esos requisitos incluyen también que se consulte a todos los interesados y, en particular, que a las víctimas se les consulte y se les permita participar en el diseño, la adopción y la implementación de un PMR. ${ }^{102}$ Estos criterios también requieren la inclusión de grupos políticamente marginados, como mujeres, niños y minorías, en el proceso de adopción y diseño de un PMR. ${ }^{103}$

La CtIDH también debe evaluar si el Estado en cuestión está implementando efectivamente un PMR adoptado, tomando todas las medidas razonables para hacerlo. ${ }^{104}$ Para evaluar esto, la Corte podría solicitar o examinar informes sobre este tema de otras organizaciones. La Corte Constitucional de Colombia, en su sentencia sobre desplazados internos emitida en 2004, hizo esto para evaluar si las políticas de derechos humanos sobre los desplazados internos en Colombia se habían aplicado efectivamente. ${ }^{105} \mathrm{Su}$ enfoque puede proporcionar orientación útil a la CtIDH sobre esta cuestión.

\footnotetext{
101 Ver informe de DE GREIFF, Pablo. Informe del Relator Especial sobre la promoción de la verdad, la justica, la reparación y las garantías de no repetición, 14 de octubre de 2014, doc. de la ONU A/69/518, párrafo 67.

102 Respecto a decisiones de la CTIDH. ver Operación Génesis vs. Colombia, sentencia de 20 de noviembre de 2013, para. 470; caso de Yarce y otros vs. Colombia, sentencia de 22 de noviembre de 2016, párrafo 326. Ver también el Conjunto de principios actualizado para la protección y la promoción de los derechos humanos mediante la lucha contra la impunidad, Principio 32; Comisión Interamericana de Derechos Humanos, Lineamientos Principales para una Política Integral de Reparaciones, OEA/Ser/L/V/II.131. Doc. 1. 19 de febrero de 2008, párrafo 13 (en adelante 'ComIDH, Lineamientos para una Política Integral de Reparaciones'); Redress, 'Articulating Minimum Standards on Reparations Programmes in Response to Mass Violations' (The Hague Redress 2014), párrafo 39.

103 ComIDH, Lineamientos para una Política Integral de Reparaciones; Organización Internacional para las Migraciones, 'Property Restitution and Compensation: Practices and Experiences of Claims Programmes', ed. Organización Internacional para las Migraciones (Ginebra, Organización Internacional para las Migraciones, 2008); Moffett, 'Transitional Justice and Reparations'.

104 Ver por ejemplo, CTIDH. caso de la Aldea de Chichupac contra Guatemala, sentencia de 30 de noviembre de 2016, párrafo 280.

105 Corte Constitucional de Colombia, sentencia T-25/04, 22 de enero de 2004, en particular secciones 7, 9 .
}

\subsection{Criterios adicionales para evaluar si un determinado PMR cumple con las obligaciones del Estado en virtud de la Convención Americana de Derechos Humanos}

En esta subsección se identifican algunos criterios adicionales que la CtIDH debe utilizar para evaluar si un determinado PMR cumple las obligaciones del Estado en virtud de la Convención Americana sobre Derechos Humanos. También se aborda la cuestión de cuál estándar de revisión debe aplicar la CtIDH para evaluar el cumplimiento de esos criterios. Varios expertos sostienen que, aparte de los requisitos generales analizados anteriormente, los Estados gozan de una amplia discreción a la hora de decidir el contenido de PMR. ${ }^{106}$ En el otro extremo del espectro, algunas ONG sostienen que los estándares aplicables a PMR y las reparaciones por parte de tribunales en casos de violaciones de los derechos humanos a gran escala deben ser esencialmente los mismos. ${ }^{107}$ Propongo un enfoque más matizado que aquellos sugeridos por estas dos posiciones. Como cuestión preliminar, vale la pena reiterar que algunos PMR adoptados en América Latina en la última década han abarcado indemnizaciones, rehabilitación, restitución y algunas formas de satisfacción directamente relacionadas con la búsqueda y el reconocimiento de la verdad.

Sostengo que la intensidad del estándar de revisión ejercido por la CtIDH debe variar de acuerdo con el componente o la forma de reparación de que se trate. Por una parte, la intensidad del estándar de revisión ejercido por la CtIDH sobre las medidas de satisfacción relacionadas directamente con la búsqueda y el reconocimiento de la verdad, y las medidas de rehabilitación relacionadas con el derecho a la salud debe ser alta o estricta. Por otro lado, la intensidad del estándar de revisión ejercido por la CtIDH en relación con indemnización, medidas de satisfacción no relacionadas con los objetivos mencionados y las medidas de rehabilitación no relacionadas con el derecho a la salud, debe ser menos estricta. La primera razón para esta distinción es

\footnotetext{
106 Oficina del Alto Comisionado de las Naciones Unidas para los Derechos Humanos, 'Rule of Law Tools for Post-Conflict States: Reparations Programmes', 7; Organización Internacional para las Migraciones, 'Property Restitution and Compensation: Practices and Experiences of Claims Programmes'; Contreras Garduno, 'Collective Reparations'.

107 Redress, 'Articulating Minimum Standards on Reparations Programmes in Response to Mass Violations'.
} 
que existen claros estándares de derecho internacional en relación con el deber del Estado de investigar las violaciones graves de los derechos humanos y revelar los resultados de dichas investigaciones, ${ }^{108}$ así como respecto al derecho a la salud. ${ }^{109}$ En cambio, con respecto a muchos aspectos de indemnización, elementos de satisfacción no relacionados con dichos objetivos y medidas de rehabilitación no vinculadas al derecho a la salud, no existen estándares jurídicos internacionales ampliamente aceptados. ${ }^{110}$ Otra razón para esta distinción es que muchas de las primeras medidas entrañan mucho menos prestaciones materiales y, por lo tanto, menos recursos financieros que la indemnización.

Aspectos importantes de PMR sobre los cuales formuladores de políticas públicas nacionales gozan de cierta latitud incluyen los siguientes: En primer lugar, quién será elegible para recibir reparaciones en virtud de un PMR, incluyendo qué tipo de violaciones o pérdidas permitirán el acceso a los beneficios y qué período se cubrirá. ${ }^{111}$ Como se examinó anteriormente, los Estados deben observar un enfoque basado en los derechos humanos al decidir sobre estos aspectos. Pablo de Greiff ha sugerido que se introduzca el requisito de que los Estados justifiquen las exclusiones del ámbito de los PMR de las categorías de víctimas que sean significativas debido a la naturaleza o la prevalencia de

\footnotetext{
108 Ver por ejemplo, Ochoa-Sánchez, The Rights of Victims in Criminal Justice Proceedings for Serious Human Rights Violations. 109 Ver Asamblea General de la Organización de las Naciones Unidas, Pacto Internacional de Derechos Económicos, Sociales y Culturales, 993; Comité de Derechos Económicos, Sociales y Culturales de la ONU [Comité DESC de la ONU], Observación General N ${ }^{\circ}$ 3: La naturaleza de las obligaciones de los Estados Partes (art. 2, párrafo 1, del Pacto), 14 de diciembre de 1990, párrafo 15; Directrices de Maastricht sobre las violaciones de los derechos económicos, sociales y culturales [Maastricht Guidelines], Maastricht, 26 de enero de 1997, http://www.refworld.org/docid/48abd5730.html. Para análisis por parte de academicos, ver LANGFORD, Malcolm (ed.) Social Rights Jurisprudence: Emerging Trends in International and Comparative Law. Cambridge: Cambridge University Press, 2009; OCHOA-SÁNCHEZ, Juan Carlos. Economic and social rights and transitional justice: A framework of analysis. Journal of Human Rights, v. 18 , n. 5 , p. $522-542,2019$.

${ }^{110}$ Ver por ejemplo, informe de DE GREIFF, Pablo. Informe del Relator Especial sobre la promoción de la verdad, la justica, la reparación y las garantías de no repetición, 14 de octubre de 2014, doc. de la ONU A/69/518; Corte Constitucional de Colombia, sentencia T-083/17, 13 de febrero de 2017, párrafo 19.2; Contreras Garduno, 'Collective Reparations', 255-256; Organización Internacional para las Migraciones, 'Property Restitution and Compensation'.

111 Ver por ejemplo, MOFFETT, L. Transitional Justice and Reparations: Remedying the Past? In: LAWTHER, C.; MOFFETT, L.; JACOBS, D. (ed.). Research Handbook on Transitional Justice. Edward Elgar, 2017. p. 13-14.
}

las violaciones. ${ }^{112}$ Estoy de acuerdo con esta recomendación realizada por de Greiff. El segundo aspecto incluido en esta categoría es qué tipos de reparaciones y beneficios proporcionará un PMR, incluida la relación entre reparaciones materiales y simbólicas, el significado de las reparaciones colectivas, ${ }^{113}$ y la relación entre reparaciones individuales y colectivas. ${ }^{114}$ Como afirma de Greiff, "reparaciones materiales y simbólicas pueden adoptar diferentes formas y combinarse de diferentes maneras", y las combinaciones tienen diferentes efectos en cuanto al número de víctimas cubiertas y necesidades de las víctimas satisfechas. ${ }^{115}$ Aparte de la prohibición de discriminación, el requisito de un proceso participativo y lo que se dijo anteriormente con respecto a las reparaciones simbólicas directamente relacionadas con la búsqueda y el reconocimiento de la verdad, parece que no hay estándares jurídicos claros sobre las determinaciones que los formuladores de políticas deben hacer sobre estas cuestiones. Sin embargo, un requisito útil que podría adoptarse sería que los Estados articulen los principios o, al menos, los fundamentos para incluir algunas reparaciones y otras no.

Otra pregunta importante que deben abordar los formuladores de políticas públicas nacionales al diseñar un PMR es dónde establecer el nivel de indemnización o compensación monetaria. Según de Greiff, "la práctica internacional [sobre este tema] en el área de reparaciones varía significativamente de un país a otro", 116 y, como se mencionó anteriormente, en la práctica

${ }^{112}$ Ver informe de DE GREIFF, Pablo. Informe del Relator Especial sobre la promoción de la verdad, la justica, la reparación y las garantías de no repetición, 14 de octubre de 2014, doc. de la ONU A/69/518. p. 17-18.

113 Contreras Garduno, 'Collective Reparations', 270 (“ 'Reparaciones colectivas' es un término al que se le ha dado diferentes significados en diferentes contextos. Ella añade: "El contenido de las reparaciones colectivas "se decide usualmente caso por caso" (p. 271). Sin embargo, Contreras identifica algunos principios sustantivos y de procedimiento que deberían informar reparaciones colectivas. Ver Contreras Garduno, 273-297, y 321.

114 Para un análisis sobre esos temas, ver CONTRERAS GARDUNO, Diana. Collective Reparations: Tensions and Dilemmas between Collective Reparations with the Individual Right to Receive Reparations'. Tesis (doctorado) Utrecht University, 2018. p. 309.

115 Ver informe de DE GREIFF, Pablo. Informe del Relator Especial sobre la promoción de la verdad, la justica, la reparación y las garantías de no repetición, 14 de octubre de 2014, doc. de la ONU A/69/518, párrafo 30.

116 DE GREIFF, Pablo. Informe del Relator Especial sobre la promoción de la verdad, la justica, la reparación y las garantías de no repetición, 14 de octubre de 2014, doc. de la ONU A/69/518, párrafo 43 . 
comparada de los PMR, "rara vez se intenta satisfacer el principio de la reparación integral". ${ }^{117}$ En cuanto al estado del derecho internacional sobre este tema, de Greiff afirma:

Si bien podría decirse que el derecho internacional deja cierto margen para la concertación de acuerdos con respecto al gran número de reparaciones que es necesario atender en casos masivos, se sigue exigiendo, como se resume en los Principios Básicos, la "[R]eparación adecuada, efectiva y rápida del daño sufrido". ${ }^{118}$

Luego, él expone algunos criterios mínimos a este respecto:

[A fin de que un PMR pueda lograr sus objetivos, tales como reconocer a las víctimas como titulares de derechos, no sólo como víctimas], la magnitud de las reparaciones tiene que ser proporcional a la gravedad de las violaciones, las consecuencias que las violaciones generaron a las víctimas, la vulnerabilidad de estas, y la intención de manifestar el compromiso de defender el principio de igualdad de derechos para todos. ${ }^{119}$

Estoy de acuerdo con de Greiff en que la discreción del Estado para fijar el nivel de la indemnización o compensación monetaria debe estar limitada por estos principios. A este respecto, cabe señalar que la CtIDH ha incluido entre los requisitos sustantivos que PMR deben cumplir los siguientes:

- El PMR relevante y sus órganos ejecutivos deben indicar claramente los criterios utilizados para establecer los montos y los conceptos de las indemnizaciones a favor de las víctimas. ${ }^{120}$

- Cualquier medida pecuniaria prevista en un PMR debe ser razonable y proporcional; ${ }^{121}$

\footnotetext{
117 DE GREIFF, Pablo. Informe del Relator Especial sobre la promoción de la verdad, la justica, la reparación y las garantías de no repetición, 14 de octubre de 2014, doc. de la ONU A/69/518, párrafo 45.

118 DE GREIFF, Pablo. Informe del Relator Especial sobre la promoción de la verdad, la justica, la reparación y las garantías de no repetición, 14 de octubre de 2014, doc. de la ONU A/69/518, párrafo 46.

119 DE GREIFF, Pablo. Informe del Relator Especial sobre la promoción de la verdad, la justica, la reparación y las garantías de no repetición, 14 de octubre de 2014, doc. de la ONU A/69/518, párrafo 47. Ver también Redress, 'Articulating Minimum Standards on Reparations Programmes in Response to Mass Violations', párrafo 71.

${ }^{120}$ CTIDH. caso de la Aldea de Chichupac contra Guatemala, sentencia de 30 noviembre de 2016, párrafo 280.

121 CTIDH. Operación Génesis vs. Colombia, sentencia de 20 de noviem-
}

- " $[\mathrm{U}] \mathrm{n}$ criterio de justicia respecto a la reparación pecuniaria debe involucrar aspectos que, en el contexto específico, no resulten ilusorios o irrisorios y permitan una contribución real para que la víctima enfrente las consecuencias negativas que dejaron las violaciones de derechos humanos en su vida. ${ }^{122}$

- PMR deben dar razones para proveer reparaciones por grupo familiar y no en forma individual, y el tipo de criterios de distribución entre miembros de una familia (órdenes sucesorales o porcentajes) ..."123

En conclusión, el análisis anterior demuestra que varios estándares internacionales de derechos humanos se aplican a los PMR, incluidos algunos específicos que varían según la forma de reparación. Por consiguiente, es inexacta la conclusión de algunos expertos y organizaciones internacionales de que, aparte de algunos requisitos generales, los Estados gozan de amplia discreción al decidir el contenido de los PMR. ${ }^{124}$

Hay dos requisitos adicionales que la $\mathrm{CtIDH}$ debe evaluar para decidir si se remite a un determinado PMR en su decisión de reparaciones en un caso determinado. En primer lugar, los beneficios establecidos en el PMR en cuestión deben estar relacionados con las violaciones de los derechos humanos establecidas en la sentencia de la CtIDH. ${ }^{125}$ El segundo requisito es que el Estado respectivo mencione el PMR específico en los procedimientos ante la CtIDH dentro de los plazos previstos para esto. ${ }^{126}$

bre de 2013, párrafo 470; CTIDH. caso de Yarce y otros vs. Colombia, sentencia de 22 de noviembre de 2016, párrafo 326.

122 CTIDH. Operación Génesis vs. Colombia, sentencia de 20 de noviembre de 2013, párrafo 471.

123 CTIDH. Operación Génesis vs. Colombia, sentencia de 20 de noviembre de 2013, párrafo 470; CTIDH. caso de Yarce y otros vs. Colombia, sentencia de 22 de noviembre de 2016, párrafo 326.

124 Ver por ejemplo, Oficina del Alto Comisionado de las Naciones Unidas para los Derechos Humanos, 'Rule of Law Tools for PostConflict States: Reparations Programmes’, 7; Organización International para las Migraciones, 'Property Restitution and Compensation'; Contreras Garduno, 'Collective Reparations'.

${ }^{125}$ Ver por ejemplo, CTIDH. caso de la Aldea de Chichupac contra Guatemala, sentencia de 30 noviembre de 2016, párrafo 280.

126 CTIDH. caso Rodríguez Vera y otros (los desaparecidos del Palacio de Justicia) vs. Colombia, sentencia de 14 de noviembre de 2014, párrafo 551. 


\section{Conclusiones}

La presente investigación ha aportado nuevas perspectivas a la pregunta: ¿qué tipo de control judicial debe ejercer la CtIDH en casos en contra de un Estado en el cual se ha implementado un PMR? Particularmente, a la pregunta: ¿qué tipo de ordenes de reparaciones debe adoptar esta corte en este tipo de casos? Primero, el análisis presentado arriba ha demostrado que el punto de vista según el cual la CtIDH debe limitarse a un enfoque de caso individual en sus órdenes de reparaciones en los casos examinados, es injustificado desde una perspectiva jurídica, analítica y normativa. La práctica internacional en materia de derechos humanos, la propia jurisprudencia de la CtIDH y la práctica constitucional comparada en materia de derechos fundamentales respecto a formas generales de control judicial por parte de tribunales de derecho público, proveen los principales fundamentos jurídicos de esa conclusión. ${ }^{127}$

Además, este artículo propone un enfoque alternativo al de caso individual y a la deferencia casi incondicional a PMR por parte de la CtIDH. Propone un enfoque en el que la CtIDH examina las características de los PMR aplicables a los casos individuales presentados ante este Tribunal. Específicamente, la CtIDH debe evaluar primero si un determinado PMR cumple con las obligaciones del Estado en virtud de la Convención Americana de Derechos Humanos. En segundo lugar, la CtIDH debe examinar si se cumplen los requisitos adicionales para remitirse a un PMR específico en su decisión sobre reparaciones. En este estudio se han examinado los diversos criterios que la CtIDH debe aplicar cuando haga lo primero, incluidos los que se aplican a PMR en su conjunto y otros criterios más específicos, que varían según la forma de reparación. En este trabajo también se ha analizado la intensidad del estándar de revisión que debe ejercer la CtIDH en relación con las principales formas de reparación.

La práctica internacional en materia de derechos humanos, la propia jurisprudencia de la CtIDH y la práctica constitucional comparada proveen las bases del enfoque propuesto. Estas demuestran que la CtIDH puede ejercer algunas formas generales de control judicial. ${ }^{128}$

\footnotetext{
127 Ver arriba la sección denominada 'Un Enfoque de caso individual: un análisis crítico', subsección 'Limitarse a un enfoque de caso individual en los casos estudiados es injustificado teniendo en cuenta los poderes de la CtIDH'.

${ }^{128}$ Ver arriba la sección denominada 'Un Enfoque de caso individ-
}

Además, la CtIDH ejercería en estos casos una forma general de control judicial relativamente limitada. Primero, existiría una política nacional de reparaciones, es decir, un PMR en el país en cuestión. Segundo, como se analizó arriba, el control por parte de la CtIDH respecto a algunos aspectos de un PMR específico sería probablemente de baja intensidad. Adicionalmente, el enfoque propuesto toma en consideración los principales aspectos de la cuestión analizada mejor que otras posiciones que han sido sugeridas. Este enfoque preservaría el rol de supervisión de la CtIDH en materia de reparaciones y, lo que es más importante, los PMR se someterían a control judicial por parte de esta corte. En particular, este enfoque garantizaría que la CtIDH evalúe si un determinado PMR cumpla con estándares internacionales aplicables a esos programas, cubriendo elementos sustantivos y elementos relacionados con el proceso. También garantizaría otros objetivos del SIDH, como la protección efectiva de los derechos humanos, ${ }^{129}$ el cual es razonable afirmar que se aplica a todas las víctimas en la misma situación en un país determinado. A este respecto, el enfoque propuesto garantizaría la coherencia de las reparaciones concedidas y un trato en condiciones de igualdad a las víctimas de violaciones de los derechos humanos en la misma situación en los países involucrados. Esto sería muy importante debido a las condiciones que suelen prevalecer en los entornos de transición, en particular el número considerable de víctimas involucradas y los recursos limitados de que disponen los Estados miembros del SIDH para satisfacer las numerosas necesidades de estas sociedades.

Finalmente, este artículo contribuye al análisis de temas más amplios de mucha importancia, tales como las condiciones bajo las cuales la $\mathrm{CtIDH}$ puede ejercer formas generales de control judicial. Esta investigación hizo esto teniendo en cuenta aportes analíticos y desarrollos recientes en la práctica internacional en derechos humanos y en la adjudicación comparada en materia de derechos fundamentales en esta materia.

ual: un análisis crítico', subsección 'Limitarse a un enfoque de caso individual en los casos estudiados es injustificado teniendo en cuenta los poderes de la CtIDH'.

129 Ver por ejemplo, CTIDH. el Derecho a la Información sobre la Asistencia Consular, Opinión Consultiva OC-16/99 de 1 de octubre de 1999, Serie A No. 16, párrafo 58. 


\section{Referencias}

ANTKOWIAK, Thomas M. 'Remedial Approaches to Human Rights Violations: The Inter-American Court of Human Rights and Beyond'. Columbia Journal of Transnational Law, v. 46, n. 2, 2008.

ASAMBLEA GENERAL DE LA ONU. Principios $y$ directrices básicos sobre el derecho de las víctimas de violaciones manifiestas de las normas internacionales de derechos bumanos $y$ de violaciones graves del derecho internacional humanitario a interponer recursos y obtener reparaciones, Res. 60/147, 16 de diciembre 2005, doc. de la ONU A/ RES/60/147.

ATTANASIO, David L. 'Extraordinary Reparations, Legitimacy, and the Inter-American Court. University of Pennsylvania Journal of International Law, v. 37, n. 3, p. 81371, 2016.

BILCHITZ, David. 'Constitutionalism, the Global South, and Economic Justice', en Constitutionalism of the Global South: The Activist Tribunals of India, South Africa, and Colombia, ed. Daniel Bonilla Maldonado. Cambridge: Cambridge University Press, 2013.

BOLIVAR J., Aura Patricia. Programas Administrativos de Reparación: El Caso Colombiano en Perspectiva' Tesis (Magister en derecho) Universidad Nacional de Colombia, 2012.

BORnEMAN, John. Political Crime and the Memory of Loss. Indiana University Press, 2011.

CAROZZA, Paolo. 'Subsidiarity as a Structural Principle of International Human Rights Law'. American Journal of International Law, v. 97, p. 78-79, 2003.

CEDAW. Recomendación General No. 35 sobre violencia de género contra las mujeres, 26 de julio de 2017, CEDAW/C/ GC/35.

CENTRO INTERNACIONAL PARA LA JUSTICIA TRANSICIONAL. 'Qué es la justicia transicional?', en Fichas ed. Centro International para la Justicia Transicional (Nueva York, 2009).

COMISIÓN INTERAMERICANA DE DERECHOS HUMANOS. Lineamientos Principales para una Politica Integral de Reparaciones, OEA/Ser/L/V/II.131. Doc. 1.19 de febrero de 2008.

COMITÉ DE DERECHOS ECONÓMICOS. Sociales y Culturales de la ONU [Comité DESC de la ONU], Observación General No 3: La naturaleza de las obliga- ciones de los Estados Partes (art. 2, párrafo 1, del Pacto), 14 de diciembre de 1990.

CONGRESO DE COLOMBIA. Ley de Victimas y Restitución de Tierras, Ley 1448/2011. Disponible en: https:/ / www.unidadvictimas.gov.co/sites/default/files/documentosbiblioteca/ley-1448-de-2011.pdf

CONTESSE, Jorge. 'Contestation and Deference in the Inter-American Human Rights System'. Law and Contemporary Problems, v. 79, 2016.

CONTRERAS GARDUNO, Diana. Collective Reparations: Tensions and Dilemmas between Collective Reparations with the Individual Right to Receive Reparations'. Tesis (doctorado) Utrecht University, 2018.

CORNEJO CHAVEZ, Leiry. 'New Remedial Responses in the Practice of Regional Human Rights Courts: Purposes Beyond Compensation'. International Journal of Constitutional Law, v. 15, n. 2, 2017.

CORREA, Cristian. 'Reparations for Victims of Massive Crimes. Making Concrete a Message of Inclusion'. En: Victimological Approaches to International Crimes: Africa. LETSCHERT, Rianne et al. (ed.). Cambridge: Antwerp and Portland: Intersentia, 2011. p. 203.

CORTE CONSTITUCIONAL DE COLOMBIA. sentencia T-083/17, 13 de febrero de 2017.

CORTE CONSTITUCIONAL DE COLOMBIA. sentencia T-25/04, 22 de enero de 2004.

CORTE INTERAMERICANA DE DERECHOS HUMANOS. ('CtIDH'). Velásquez Rodriguez contra Honduras, sentencia de fondo, 29 de julio de 1988, Serie C No. 4.

CORTE INTERNACIONAL DE JUSTICIA. LaGrand (Alemania c. Los Estados Unidos), fondo, sentencia de 27 de junio de 2001.

CRAWFORD, James. (ed.) The International Law Commission's Articles on State Responsibility: Introduction, Text, and Commentaries. Cambridge: Cambridge University Press, 2002.

CTIDH. caso de Almonacid-Arellano y otros contra Chile, Sentencia de objeciones preliminares, fondo, reparaciones y costos, 26 de septiembre de 2006, Serie C No. 154.

CTIDH. caso de la Aldea de Chichupac y comunidades vecinas del municipio de Rabinal contra Guatemala, sentencia de excepción preliminar, fondo, reparaciones y costas, 30 de noviembre de 2016. 
CTIDH. caso de la masacre de Plan de Sánchez. contra Guatemala, sentencia de fondo, 29 de abril de 2004.

CTIDH. caso de la masacre de Plan de Sánchez contra Guatemala, sentencia de reparaciones, 19 de noviembre de 2004.

CTIDH. caso de la Vereda La Esperanza vs. Colombia, 31 de agosto de 2017.

CTIDH. caso de Las Palmeras contra Colombia, Sentencia de reparaciones, 26 de noviembre de 2002, Serie C No. 96.

CTIDH. caso de Manuel Cepeda Vargas vs. Colombia, sentencia de 26 de mayo de 2010.

CTIDH. caso de Tenorio Roca y otros vs. Peru, sentencia de 22 de junio de 2016.

CTIDH. caso de Yarce y otros vs. Colombia, sentencia de objeciones preliminares, fondo, reparaciones y costos, 22 de noviembre de 2016.

CTIDH. caso García Lucero y otros vs. Chile, sentencia de 28 de Agosto de 2013.

CTIDH. caso Godinez Crur, sentencia de fondo, 20 de enero de 1989, Serie C No. 5.

CTIDH. caso masacres de Rio Negro vs. Guatemala, sentencia de excepción preliminar, fondo, reparaciones y costas, 4 de Septiembre de 2012.

CTIDH. caso Rodríguez. Vera y otros (los desaparecidos del Palacio de Justicia) vs. Colombia, sentencia de 14 de noviembre de 2014.

CTIDH. Comunidades afrodescendientes desplazadas de la cuenca del rio Cacarica (Operación Génesis) vs. Colombia, sentencia de 22 de noviembre de 2016.

CTIDH. el Caracazo contra Venezuela, sentencia de reparaciones. 29 de agosto de 2002, Serie C No. 95.

CTIDH. el Derecho a la Información sobre la Asistencia Consular, Opinión Consultiva OC-16/99 de 1 de octubre de 1999, Serie A No. 16, párrafo 58.

CTIDH. Juan Humberto Sánchez vs. Honduras, sentencia de 7 de junio de 2003.

CTIDH. Trujillo-Oroza contra Bolivia, sentencia de reparaciones, 27 de febrero de 2002, Serie C No. 92.

CTIDH. Velasquez Rodriguez vs. Honduras, sentencia de reparaciones, 21 de julio de 1989.
DE GREIFF, Pablo. 'Justice and Reparations', en The Handbook of Reparations. Oxford: Oxford University Press, 2006.

DE GREIFF, Pablo. Informe del Relator Especial sobre la promoción de la verdad, la justica, la reparación y las garantías de no repetición, 14 de octubre de 2014, doc. de la ONU A/69/518.

GARCÍA-GODOS, Jemima. 'Victim Reparations in Transitional Justice - What is at Stake and Why'. Nordic Journal of Human Rights, v. 26, n. 2, 2008.

GARCÍA-GODOS, Jemima; SALVADÓ, Luis Raúl. Guatemala: Truth and memory on trial. En: SKAAR, E.; GARCIA-GODOS, J.; COLLINS, C. (ed.) Transitional Justice in Latin America. Londres: Routledge, 2016.

GENEVIÈVE, Lessard. 'Preventive Reparations at a Crossroads: The Inter-American Court of Human Rights and Colombia's Search for Peace'. The International Journal of Human Rights, v. 22, n. 9, 2018.

GOBIERNO DE COLOMBIA. Alegatos Finales Escritos, República de Colombia, Caso No. 12.573 "Marino López Mena y Otros- Operación Génesis".

GOBIERNO DE GUATEMALA. Comisión para el Esclarecimiento Histórico (1999). Guatemala: Memoria del Silencio, Conclusiones and Recomendaciones (Ciudad de Guatemala: Comisión para el Esclarecimiento Históri$\mathrm{co})$.

GOBIERNO DE GUATEMALA. Presidencia de la Republica, Comisión Presidencial Coordinadora de la Política del Ejecutivo en Materia de Derechos Humanos COPEDEH, Alegatos Finales del Estado de Guatemala dentro del Caso 12.599 Florencio Chitay v. Guatemala, Presentado ante la Honorable Corte Interamericana de Derechos Humanos, 3 de marzo de 2010.

GOBIERNO DE GUATEMALA. Presidencia de la Republica, COPREDEH, 21 de mayo de 2004, REDA-28-2004.

GUARNIZO, Diana. 'Guarantees of Non-Repetition and the Right to Health: Review of the Law and Evolving Practice of Judicial and Semi-Judicial Bodies at Global and Regional Levels'. Tesis (Doctorado) University of Essex, 2106.

HUNEEUS, Alexandra. 'Reforming the State from Afar: Structural Reform Litigation at the Human Rights Courts'. Yale Journal of International Law, v. 40, n. 1, p. 1-40, 2015. 
INTERNATIONAL COMMISSION OF JURISTS. Directrices de Maastricht sobre las violaciones de los derechos económicos, sociales y culturales. [Maastricht Guidelines], Maastricht, 26 de enero de 1997. Disponible en: http:// www.refworld.org/docid/48abd5730.html.

JACHTENFUCHS, Markus; KRISCH, Nico. 'Subsidiarity in Global Governance', 79 Law and Contemporary Problems, v. 79, 2016.

LANGFORD, Malcolm (ed.) Social Rights Jurisprudence: Emerging Trends in International and Comparative Law. Cambridge: Cambridge University Press, 2009.

LANGFORD, Malcolm. 'Social Rights Adjudication. An Interdisciplinary Perspective'. Tesis (Doctorado) Facultad de Derecho, Universidad de Oslo, 2014.

LONDOÑO LÁZARO, María Carmelina; HURTADO, Mónica. 'Las Garantías De No Repetición en la Práctica Judicial Interamericana y su Potencial Impacto en la Creación del Derecho Nacional'. Boletín mexicano de derecho comparado, 50, 2017.

MALAMUD-GOTI, Jaime E.; GROSMAN, Lucas Sebastián; DE GREIFF, Pablo. 'Reparations and Civil Litigation: Compensation for Human Rights Violations inTransitional Democracies'. En: DE GREIFF, Pablo. (ed.). The Handbook of Reparations. Oxford: Oxford University Press, 2006.

MANJOO, Rashida. Informe de la Relatora Especial sobre la Violencia contra la Mujer, sus causas y consecuencias, 23 abril 2010, doc. de la ONU A/HRC/14/22.

MERSKY, Marcie; ROHT-ARRIAZA, Naomi. 'Guatemala'. En: SALAZAR, Katya; ANTKOWIAK,Thomas. (ed.). Victims Unsilenced: The Inter-American Human Rights System and Transitional Justice in Latin America. Washington, DC: Due Process of Law Foundation, 2007.

MOFFETT, L. Transitional Justice and Reparations: Remedying the Past? In: LAWTHER, C.; MOFFETT, L.; JACOBS, D. (ed.). Research Handbook on Transitional Justice. Edward Elgar, 2017.

MOON, Claire. 'Who'll Pay Reparations on My Soul?'1 Compensation, Social Control and Social Suffering'. Social \& Legal Studies 21, n. 2, 2012.

OCHOA-SÁNCHEZ, Juan Carlos. Economic and social rights and transitional justice: A framework of analysis. Journal of Human Rights, v. 18, n. 5, p. 522-542, 2019).
OCHOA-SÁNCHEZ, Juan Carlos. The Rights of Victims in Criminal Justice Proceedings for Serious Human Rights Violations. Leiden: Martinus Nijhoff, 2013.

OFICINA del Alto Comisionado de las Naciones Unidas para los Derechos Humanos, 'Rule of Law Tools for Post-Conflict States: Reparations Programmes' (OADH: Geneva).

ORGANIZACIÓN INTERNACIONAL PARA LAS MIGRACIONES. 'Property Restitution and Compensation: Practices and Experiences of Claims Programmes', ed. Organización Internacional para las Migraciones. Ginebra: Organización Internacional para las Migraciones, 2008.

PASQUALUCCI, Jo M. 'Victim Reparations in the Inter-American Human Rights System: A Critical Assessment of Current Practice and Procedure'. Michigan Journal of International Law 18, 1996.

REDRESS. 'Articulating Minimum Standards on Reparations Programmes in Response to Mass Violations' (The Hague Redress 2014).

ROACH, Kent. 'The Challenges of Crafting Remedies for Violations of Socio-Economic Rights', en: LANGFORD, Malcolm. (ed.). Social Rights Jurisprudence: Emerging Trends in International and Comparative Law. Cambridge: Cambridge University Press, 2009.

RODRÍGUEZ-GARAVITO, César; RODRÍGUEZFRANCO, Diana. Juicio a La Exclusión: El Impacto de los Tribunales sobre los Derechos Sociales en el Sur Global. Buenos Aires: Siglo 21 Editora Iberoamericana, 2015.

ROHT-ARRIAZA. 'Central America, the Inter-American System, and Accountability for International Crimes'. UC Hastings Research Paper, n. 6, 2012.

RUBIO-MARÍN Ruth; SANDOVAL, Clara. 'Engendering the Reparations Jurisprudence of the InterAmerican Court of Human Rights: The Promise of the 'Cotton Field' Judgment'. Human Rights Quarterly, 33, n. 4, 2011.

SALVIOLI, Fabian. Informe del Relator Especial sobre la promoción de la verdad, la justicia, la reparación, y las garantías de no reparación, 11 de julio de 2019, documento. de la ONU A/HRC/42/45. 
SANDOVAL, Clara. 'Two Steps Forward, One Step Back: Reflections on the Jurisprudential Turn of the Inter-American Court of Human Righs on Domestic Reparation Programmes'. The International Journal of Human Rights, 22, n. 9, 2018).

TRIBUNAL PERMANENTE DE JUSTICIA INTERNACIONAL. Factory at Chorzón, Alemania c. Polonia, reclamación por indemnización, sentencia No 13, (1928) PCIJ Series A No 17, ICGJ 255 (PCIJ 1928), 13 septiembre 1928.

TSERETELI, Nino. 'Emerging doctrine of deference of the Inter-American Court of Human Rights?'. The International Journal of Human Rights, v. 20, n. 8, p. $1097-$ 1112, 2016.

TSERETELI, Nino. 'The Role of the European Court of Human Rights in Facilitating Legislative Change in Cases of Long-Term Delays in Implementation'. En: FØLLESDAL, Andreas; ULFSTEIN, Geir; SAUL, Matthew. (ed.). The International Human Rights Judiciary and National Parliaments: Europe and Beyond, Studies on Human Rights Conventions. Cambridge: Cambridge University Press, 2017.

TUSHNET, Mark. Weak Courts, Strong Rights: Judicial Review and Social Welfare Rights in Comparative Constitutional Law. Princeton: Princeton University Press, 2009. 
Para publicar na Revista de Direito Internacional, acesse o endereço eletrônico www.rdi.uniceub.br ou www.brazilianjournal.org.

Observe as normas de publicação, para facilitar e agilizar o trabalho de edição. 